\title{
Reviewing molecular adaptations of Lyme borreliosis spirochetes in the context of reproductive fitness in natural transmission cycles
}

\author{
Jean I. TSAO ${ }^{1,2 *}$ \\ ${ }^{1}$ Department of Fisheries and Wildlife, Michigan State University, East Lansing, MI 48864, USA \\ 2 Department of Large Animal Clinical Sciences, Michigan State University, East Lansing, MI 48864, USA
}

(Received 11 September 2008; accepted 15 April 2009)

\begin{abstract}
Lyme borreliosis (LB) is caused by a group of pathogenic spirochetes - most often Borrelia burgdorferi, B. afzelii, and B. garinii - that are vectored by hard ticks in the Ixodes ricinus-persulcatus complex, which feed on a variety of mammals, birds, and lizards. Although LB is one of the best-studied vector-borne zoonoses, the annual incidence in North America and Europe leads other vector-borne diseases and continues to increase. What factors make the LB system so successful, and how can researchers hope to reduce disease risk - either through vaccinating humans or reducing the risk of contacting infected ticks in nature? Discoveries of molecular interactions involved in the transmission of LB spirochetes have accelerated recently, revealing complex interactions among the spirochete-tick-vertebrate triad. These interactions involve multiple, and often redundant, pathways that reflect the evolution of general and specific mechanisms by which the spirochetes survive and reproduce. Previous reviews have focused on the molecular interactions or population biology of the system. Here molecular interactions among the LB spirochete, its vector, and vertebrate hosts are reviewed in the context of natural maintenance cycles, which represent the ecological and evolutionary contexts that shape these interactions. This holistic system approach may help researchers develop additional testable hypotheses about transmission processes, interpret laboratory results, and guide development of future LB control measures and management.
\end{abstract}

Lyme borreliosis / $\mathbf{R}_{\mathbf{0}}$ / vector-borne / tick

Table of contents

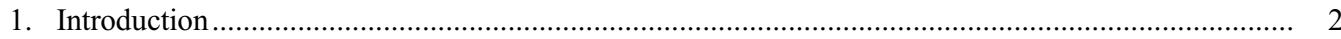

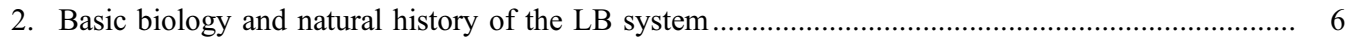

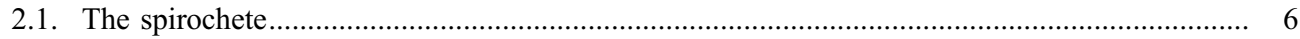

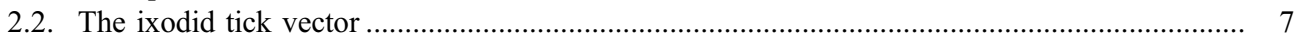

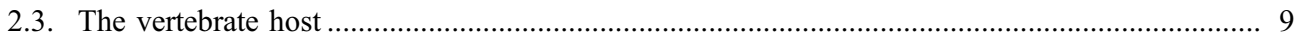

3. Reproductive fitness $\left(\mathrm{R}_{0}\right)$ as a framework to assess molecular interactions among LB

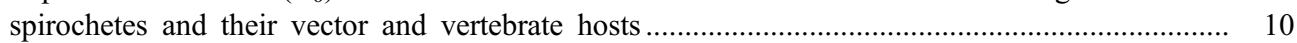

4. Interactions among LB spirochetes and their vector and vertebrate hosts...................................... 11

4.1. Vector competency and LB spirochete transmission from vertebrate to tick $\left(\beta_{\mathrm{V}-\mathrm{T}}\right) \ldots \ldots \ldots \ldots \ldots \ldots . . . . .11$

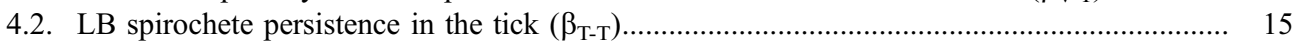

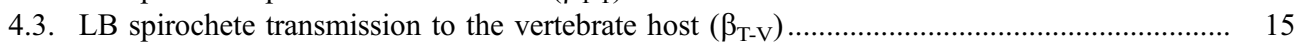

\footnotetext{
* Corresponding author: tsao@msu.edu
} 


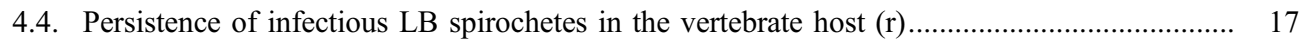

4.5. Vector-host interactions and effects on LB spirochete transmission ..................................... 20

5. Evolutionary and ecological context for adaptations of LB spirochetes........................................ 23

5.1. Generalists all around: implications for spread and impact on LB spirochete diversity ........ 23

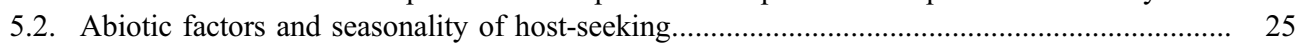

5.3. Aggregation of ticks on hosts and non-systemic transmission............................................ 26

5.4. Interactions with other pathogens and tick endosymbionts ............................................... 27

6. Discussion: incorporating an ecological and evolutionary perspective into LB research, management and control

\section{INTRODUCTION}

Lyme borreliosis (LB) is an emerging vector-borne zoonotic disease of temperate climates, with a current distribution spanning North America and Eurasia. It is caused by Borrelia burgdorferi and closely related spirochetes that are vectored by hard ticks in the Ixodes ricinus-persulcatus complex ${ }^{1}$ [65], which feed on a variety of mammals, birds, and lizards. Although LB is one of the beststudied vector-borne zoonoses, with research spanning molecular to landscape levels, the annual incidence in North America and Europe leads other vector-borne diseases and continues to increase [11].

LB normally is highly treatable; however, the treatment cost and loss of productive workdays are not trivial [302]; furthermore, for some people, there are concerns of chronic disease, although the nature of chronic LB is hotly debated [62]. An effective LB vaccine is available for canines [143], but not for people [295]. Effective acaricides are available, but their application often is undesired, cost-prohibitive, or infeasible [87, 206]. Similarly, host-targeted wildlife acaricides and repellants (like those used on domestic companion animals) have been developed for deer and rodents, but are not yet widely used $[36,56,254]$. Some communities have decided to cull deer to reduce tick populations, but currently, that is not a generally acceptable or feasible solution [217]. Thus, public health agencies strive to improve

\footnotetext{
${ }^{1}$ Keirans J., Oliver J. Jr., Needham G., The Ixodes ricinus/persulcatus complex defined, Proc. First Intl. Conference on Tick-borne Pathogens at the Vector-Host Interface, St. Paul, 1992, 302 p.
}

understanding of vector distributions [53], to promote awareness, and to prescribe behaviors to reduce risk of exposure, infection and disease [207]. In the USA, populations of I. scapularis, the vector responsible for the majority of human and canine disease, have been increasing locally and are spreading regionally, contributing to the on-going emergence of LB $[11,53]$. Anticipating that demand for a human vaccine consequently will rise, researchers continue to hunt for vulnerable molecular targets of $B$. burgdorferi and the vector. There is also ongoing research to develop host-targeted and wildlife vaccines as an additional tool for integrated disease management [40, 47, 278].

Discoveries of molecular interactions involved in LB spirochete transmission have accelerated recently due to a synergy of knowledge and tools, including the publication of several Lyme spirochete and vector species genomes, advances in genetic and immunological high-throughput techniques and molecular manipulations, and experience in executing transmission experiments in well-characterized laboratory animal models. Studies reveal complex interactions among the spirochete, tick, and vertebrate triad that involve multiple, and often redundant, pathways. These reflect, in part, the evolution of general and specific mechanisms by which the spirochetes survive and reproduce.

What factors make the LB system so successful? How can researchers hope to reduce disease risk either through vaccinating humans or reducing the probability of contacting infected ticks in nature? A succession of reviews have been written about the molecular mechanisms by which $B$. burgdorferi interacts with its vertebrate and tick hosts (e.g. [33, 64, 


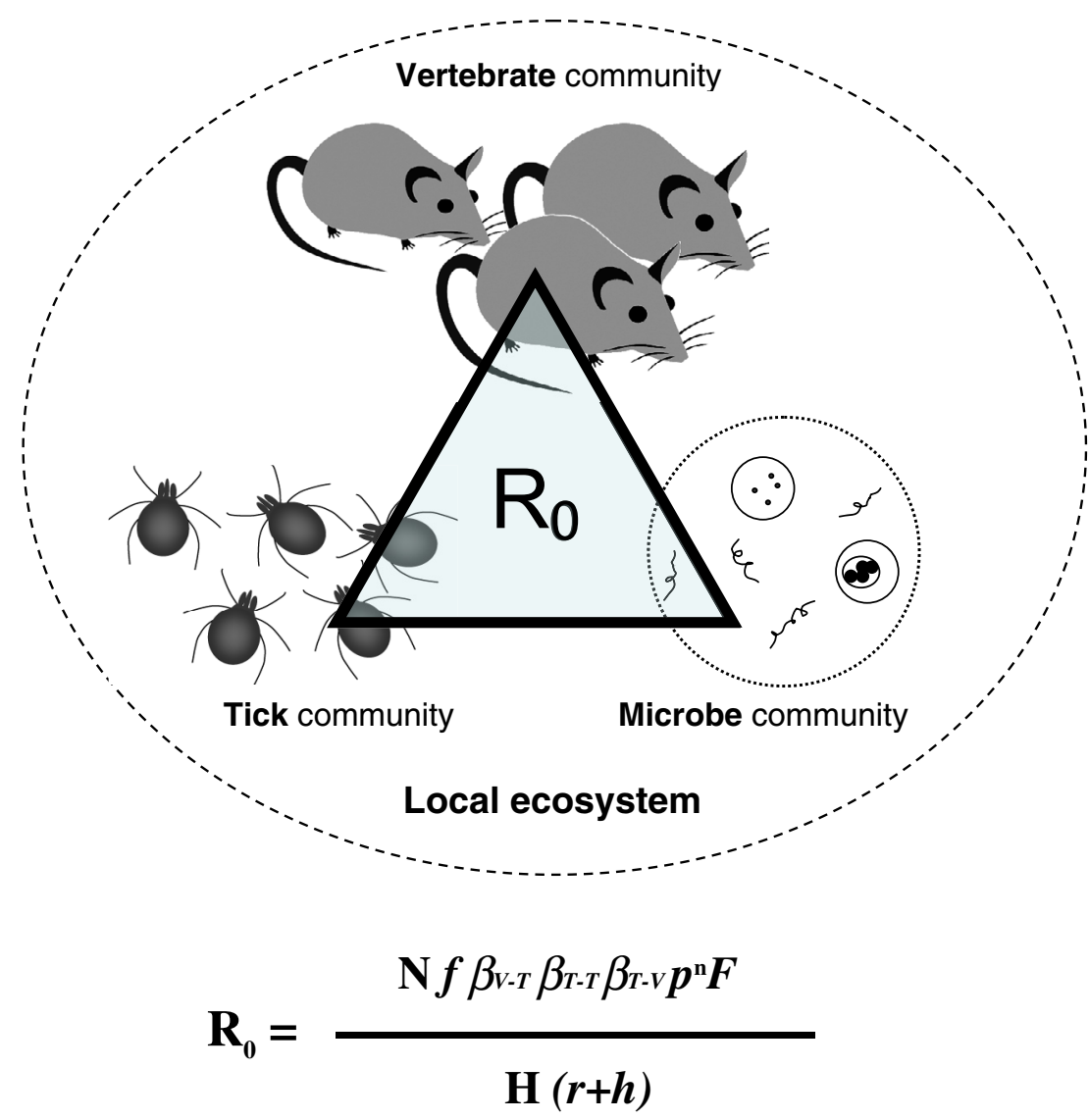

Figure 1. $\mathrm{R}_{0}$ and the vector-pathogen-host triangle within the environment. Interactions amongst all three organisms shape the adaptations of LB spirochetes for vectorial transmission. At the heart of the triangle is $\mathrm{R}_{0}$, the reproductive number for LB spirochetes, which is a measure of its fitness in a given environment, where $\mathrm{N} / \mathrm{H}$ is the ratio of ticks to vertebrate hosts; $f$ is the combined probability of a tick contacting and feeding successfully on a host; $\beta_{\mathrm{V}-\mathrm{T}}$ is the transmission coefficient for LB spirochetes from an infected vertebrate to a tick; $\beta_{\mathrm{T}-\mathrm{T}}$ is the transstadial transmission coefficient through the molt from one life stage to the next one; $\beta_{\mathrm{T}-\mathrm{V}}$ is the transmission coefficient for LB spirochetes from an infected tick to a vertebrate; $p$ is the tick's daily survival probability; $n$ is the extrinsic incubation period; $F$ is the vector's fecundity; $r$ is the host's daily recovery rate; and $h$ is the host's daily mortality rate. See Appendix for further explanation.

104, 124, 151, 251, 276]). To a lesser extent, the evolutionary ecology of LB spirochetes has been reviewed in regard to its potential impacts on LB epidemiology [125]. Here, the goal is to integrate these two perspectives: to review the molecular interactions among LB spirochetes, its vectors, and vertebrate hosts in the context of natural maintenance cycles, which represent ecological and evolutionary contexts that shape the former. Furthermore, it has been shown that the genetic diversity of LB spirochetes, which manifests as trait variation, has epidemiological and ecological consequences as well [125, 294]. Thus, a holistic system approach to examining the molecular traits of LB spirochetes may help researchers develop additional testable hypotheses about transmission processes, interpret laboratory results, and guide development of future LB control and management approaches. 
Table I. Species comprising and/or potentially influencing the Lyme borreliosis biocoenosis.

\section{Vertebrate Community}

Competent reservoir hosts

Less competent reservoir hosts

Incompetent hosts

\section{Tick Community}

Bridge vectors

Cryptic vectors, sometimes sympatric with vectors

Incompetent ticks, often sympatric with vectors
Mammals - Apodemus flavicollis (O), Blarina brevicauda $(\mathrm{N})$, Lepus spp. (N, O), Microtus pennsylvanicus (N), Myodes [formerly Clethrionomys] glareolus (O),

Peromyscus leucopus (N), Sciurus carolinensis, (N,O), S. griseus (N), S. vulgaris (O), Sorex spp. (O, N), Tamias spp. (N, O)

Birds - Fratercula arctica $(\mathrm{O}, \mathrm{N})$, Turdus migratorius $(\mathrm{N})$, T. merula $(\mathrm{O})$,

T. philomelos $(\mathrm{O})$, Phasianus colchicus $(\mathrm{O})$

Lizards - Eumeces inexpectatus (N), Lacerta agilis (O),

L. viridis $(\mathrm{O})$, Podarcis muralis $(\mathrm{O})$

Mammals - Canis latrans $(\mathrm{N})$, Didelphis virgianus $(\mathrm{N})$, Procyon lotor $(\mathrm{N})$, S. carolinensis $(\mathrm{N})$

Birds - Cardinalis cardinalis $(\mathrm{N})$, Melospiza melodia $(\mathrm{N})$

Lizards - Anolis carolinensis (N), Sceloporus undulatus (N)

Mammals - Capreolus capreolus (O), Cervus elaphus $(\mathrm{O})$,

Dama dama $(\mathrm{N})$, Odocoileus hemionus $(\mathrm{N})$, Odocoileus virginianus $(\mathrm{N})$

Birds - Dumatella carolinensis ${ }^{1}(\mathrm{~N})$, Pipilo erythrophthalmus $(\mathrm{N})$, Toxostoma rufum $(\mathrm{N})$

Lizards - Elgaria multicarinata $(\mathrm{N})$, Sceloporus occidentalis $(\mathrm{N})$

Ixodes pacificus $(\mathrm{N})$, I. persulcatus $(\mathrm{O})$, I. ricinus $(\mathrm{O})$, I. scapularis $(\mathrm{N})$

I. affinis $(\mathrm{N})$, I. angustus $(\mathrm{N})$, I. dentatus $(\mathrm{N})$, I. hexagonus $(\mathrm{O})$, I. jellisoni $(\mathrm{N})$, I. minor $(\mathrm{N})$, I. muris $(\mathrm{N})$, I. spinipalpis $(\mathrm{N})$, I. uriae $(\mathrm{O}, \mathrm{N})$

Amblyomma americanum (N), Dermacentor andersoni $(\mathrm{N})$, D. occidentalis $(\mathrm{N})$,

D. variabilis $(\mathrm{N})$, I. cookei $(\mathrm{N})$, I. tranguliceps $(\mathrm{O})$ 
Table I. Continued.

\section{Microbe Community \\ Lyme borreliosis group spirochetes \\ (species of known pathogenicity)}

Relapsing fever spirochetes

Other microbes
Borrelia andersonii $(\mathrm{N})$, B. afzelii $(\mathrm{O})$, B. bisettii $(\mathrm{N})$, B. burgdorferi $(\mathrm{O}, \mathrm{N})$ B. californiensis $(\mathrm{N}), B$. carolinensis $(\mathrm{N})$, B. garinii $(\mathrm{O})^{2}, B$. japonica $(\mathrm{O})$, B. lusitaniae $(\mathrm{O})$, B. sinica $(\mathrm{O})$, B. spielmanii $(\mathrm{O})$, B. tanukii $(\mathrm{O})$, B turdi $(\mathrm{O})$, B. valaisiana $(\mathrm{O})$, B. yantgze $(\mathrm{O})$

B. miyamotoi $(\mathrm{O}, \mathrm{N})$, “B. davisii" $(\mathrm{N})$, B. lonestari $(\mathrm{N})$

Anaplasma phagocytophilum $(\mathrm{O}, \mathrm{N})$, Babesia spp. $(\mathrm{O}, \mathrm{N})$, Bartonella spp. $(\mathrm{O}, \mathrm{N})$, Rickettsia spp. $(\mathrm{O}, \mathrm{N})$,

flaviviruses $(\mathrm{O}, \mathrm{N})$ including tick-borne encephalitis virus $(\mathrm{O})$,

louping ill virus $(\mathrm{O})$, and deertick virus $(\mathrm{N})$, known and

unknown endosymbionts $(\mathrm{O}, \mathrm{N})$

This list of species is meant to be informative and is not comprehensive. Host and tick species were selected based on common occurrence in the literature at the time of writing. Characterization of species as competent, less competent, or even incompetent is meant to illustrate the range of variation among taxa, but is not meant to be prescriptive, as competence may depend on the local community of hosts, vectors, and microbes. Microbial taxa include those known to share tick or vertebrate hosts. The geographic distributions of species are indicated generally as New $(\mathrm{N})$ or Old $(\mathrm{O})$ World. References for vertebrate hosts: [43, 46, 79, 81, 96, $97,107,121,127,129,133,141,147,154,156,157,188,231,234,266,268,269,271,282]$. References for vectors: [26, 59] and references therein, [91] and references therein, $[155,201,238]$. References for microbial community: $[13,15,20,21,31,34,39,41,44,45,68,74,93,113,131,136,145,149,152,163$, 167, 196, 199, 208-210, 232, 235, 237, 240, 241, 247, 259, 267, 272, 285].

${ }^{1}$ Contrary to a previous study [81], Ginsberg et al. [153] recently found xenodiagnostic larvae to be infected at a low level; the assay used, however, may not differentiate between B. burgdorferi and B. miyamotoi.

${ }^{2}$ While the epidemiological significance of $B$. garinii is greatest in Eurasia, it is associated with pelagic seabirds species that have a circumpolar distribution that even range into the southern hemisphere. In 2006 B. garinii was reported in I. uriae ticks collected off the coast of Newfoundland, making it the second LB spirochete species to be found in both North America and Eurasia [252]. 
This review begins with an overview of the biology and natural history of the LB system (Section 2) and then introduces the concept of $\mathrm{R}_{0}$, which provides the theoretical framework within which to consider spirochete-tick-host interactions with regard to spirochete reproductive success (Section 3). Getting to the heart of the article, the review showcases several spirochete-tick-host interactions at the molecular interface that appear to be key for persistence of LB spirochetes in nature (Section 4). The review then describes environmental, ecological, and evolutionary factors that may influence and shape the nature and evolution of those molecular interactions (Section 5). Finally, the review concludes with a brief discussion of questions for which a population perspective may help guide current and future research (Section 6). I aim to be illustrative, not comprehensive - furthermore, while I include examples from various systems, because of my particular experiences, the review primarily highlights the LB system in the northeastern USA.

\section{BASIC BIOLOGY AND NATURAL HISTORY OF THE LB SYSTEM}

Several species of spirochetes, ticks, and vertebrates participate in LB spirochete systems, which are found predominantly in northern temperate regions (Fig. 1, Tab. I). Basic biologies of each of the three suites of organisms in relation to their maintenance of LB spirochetes in nature are reviewed. It is important to remember that their ecologies, including their interspecific interactions, are influenced by local and regional ecosystems including the abiotic factors.

\subsection{The spirochete}

Spirochetes are an ancient monophyletic group of bacteria - including both parasitic and free-living forms - that have a characteristic spiral shape and a unique mode of motility that enables them to swim efficiently through highly viscous media, such as connective tissue, where the movement of other bacteria is reduced or inhibited $[38,142]$. The shape and motility of
LB spirochetes are provided by flagella located in the periplasmic space between the peptidoglycan layer and the outer membrane [165]. Several perplasmic flagella (PF) attach to each end of the cell, wrap around the cell cylinder and overlap at the cell center, giving the bacterium its characteristic flat-wave shape. The asymmetric rotation of the PF bundles generates backward moving waves along the cell body that propel it forward. Video footage of the dissemination of live $B$. burgdorferi spirochetes within a living mouse reveals how several types of motion are used to navigate through endothelial tissues [164].

Since $B$. burgdorferi was first described in 1984 , a few years after an unusual outbreak of juvenile arthritis in Lyme, Connecticut, USA [258], more than a dozen closely related spirochetes have been identified worldwide, and more undoubtedly will be described [235]. Four species are known to cause human LB: B. burgdorferi, B. afzelii, B. garinii, and B. spielmanii [235]. Most of the known species diversity of LB spirochetes occurs in Europe, suggesting an Old World center of origin for the group. Currently B. burgdorferi is the only LB spirochete that is endemic to both North America and Europe, although B. garinii was recently discovered in $I$. uriae ticks found along the coast of Newfoundland (North America) [252]. B. burgdorferi is more common in North America than in Europe where B. afzelii and $B$. garinii predominate [124]. While earlier studies suggested that $B$. burgdorferi was more genetically diverse in North America [67, 225], recent multilocus sequence typing analyses $[150,214]$ have produced contrary results and support a European origin for this spirochete, which is more consistent with the hypothesized evolutionary history of the LB group. An alternative, but not mutually exclusive, hypothesis is that recent migration of some genotypes from North America to Europe may have occurred [214].

\subsubsection{LB spirochetal genomes}

In order for LB spirochetes to adapt to their environments, heritable genetic variation among traits must exist. Mutation and recombination 
rates, as well as the mechanisms by which genetic variants are created, are fundamental to the nature and rate of adaptive change and evolution. Thus, considering the architecture of LB spirochetal genomes can provide insight into the processes that give rise to adaptations. Additionally, comparisons among genomes can further illuminate the evolution of this group of spirochetes.

A striking feature of the published genomes of $B$. burgdorferi, B. garinii, and B. afzelii is their highly segmented organization $[73,84$, 85]. At approximately $1.5 \mathrm{Mb}, \mathrm{LB}$ spirochete genomes are comprised of one linear chromosome and many linear and circular plasmids, more than any other characterized bacterium. A high degree of homology among species exists at the chromosome level, but the plasmid portion of the genomes varies greatly even among strains within a species [84, 85, 109, 274]. Plasmids encode $\sim 40 \%, 29 \%$, and $36 \%$ of the genomes of the B31 strain of $B$. burgdorferi, PBi strain of $B$. garinii, and Pko strain of B. afzelii, respectively [85]. Furthermore, the presence of many gene duplications, pseudogenes, and the partial or whole absence of plasmids suggest that evolution among the plasmid portion of the genome is more dynamic than on the chromosome [236, 261].

The chromosome contains many homologs of known genes, including housekeeping genes $[73,236,261]$. In contrast, many plasmidencoded genes require experiments to infer their functions. For example, researchers have observed the phenotypic effects of the loss of partial and whole plasmids on the infectivity for vertebrates [261]. Many genes encoding putative lipoproteins are found on the chromosome, with even more on plasmids [37]. Recent studies suggest that many plasmid genes encode proteins important to spirochete reproduction, infection, transmission, and persistence in various vertebrate hosts [85, 92, 244, 298].

\subsubsection{LB spirochete persistence in I. ricinus- persulcatus ticks in nature}

LB spirochetes are maintained in nature by I. ricinus-persulcatus complex ticks, although other ixodid ticks participate in enzootic maintenance (see below) [296]. I. ricinuspersulcatus complex ticks have three postembryonic developmental stages - larva, nymph and adult. With the exception of the adult male, each life stage requires a blood meal from a vertebrate host. LB spirochetes primarily are maintained in nature through horizontal transmission between their tick and vertebrate hosts (Fig. 2). A naïve larva first becomes infected through an infectious blood meal. The spirochete is maintained transstadially (i.e. through the molt), and the transmission cycle is completed when infected nymphs transmit LB spirochetes back to other reservoir hosts during their blood meals. Transstadial transmission requires that spirochetes survive a number of physiological events, including blood meal digestion and molting.

It is believed that transmission from infected nymphal ticks of one cohort to larval ticks of another via reservoir hosts is largely reponsible for LB spirochete maintenance in nature. This is hypothesized because adult ticks usually feed on deer or other ungulates, which are incompetent hosts, and because the ecological importance of vertical, or transovarial, transmission ranges from undetermined (I. ricinus systems) to negligible (I. scapularis and I. pacificus-driven systems) [198]. Because of the high fecundity of female ticks, however, the contribution of vertical transmission to LB spirochete persistence may be important in some LB systems [219].

Non-systemic, or co-feeding, transmission represents a third potential route by which infected ticks may infect naïve ticks [220]. In non-systemic transmission, spirochetes recently deposited into the feeding lesion by an infected tick may infect another closely, or sometimes subsequently, feeding tick. Non-systemic transmission has been demonstrated experimentally in the laboratory, but its relative importance in nature, especially in the presence of systemic transmission, is unknown [180, 197, 205].

\subsection{The ixodid tick vector}

Several tick species may vector LB spirochetes in nature. Bridge vectors are those that feed on both wildlife and humans and consequently, can present a direct zoonotic risk. In North America, the bridge vectors are 


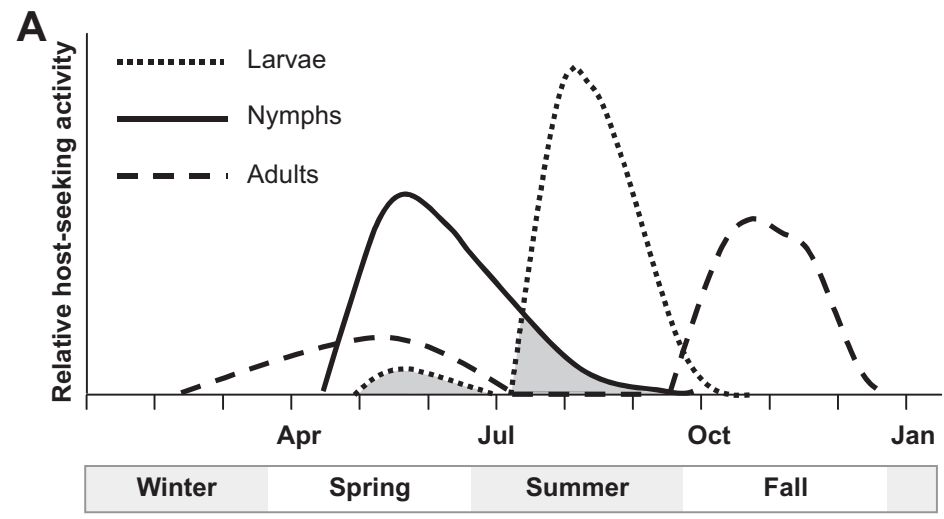

B

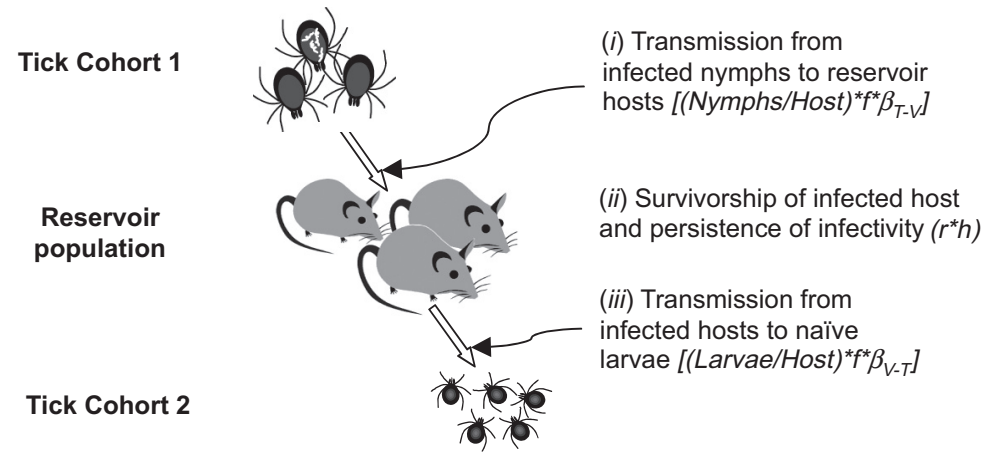

\begin{tabular}{|llll|} 
Winter & Spring & Summer & Fall \\
\hline
\end{tabular}

C

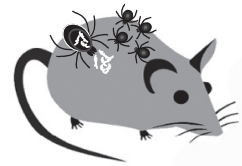

Simultaneous (true co-feeding)

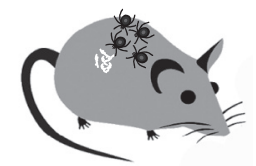

Within a few days of nymphal feeding

Figure 2. (A) I. scapularis phenology in southern New England, Northeastern USA, based on Fish (1993) [66] (Westchester County, NY, USA) and Tsao unpublished data (New Haven County, CT, USA). (B) Seasonal timing of horizontal pathogen transmission from nymphs (cohort 1) to larvae (cohort 2) via a shared reservoir hosts. Also noted are the relevant parameters influencing spirochete transmission as modified from Randolph and Craine [219]; see text for explanation. (C) Non-systemic transmission may potentially occur when naïve larvae feed either simultaneously with or very soon after an infected nymph has fed; larvae must feed near the source feeding lesion. Shaded areas of the larval and nymphal hostseeking phenology curves in (A) indicate the period over which non-systematic transmission may occur.

I. scapularis (midwestern and eastern) and I. pacificus (western). In Europe, the bridge vector is I. ricinus, whose northeastern popula- tions overlap with that of $I$. persulcatus, a bridge vector that extends far into Asia. In contrast to bridge vectors, other ticks, both within 
and outside the I. ricinus-persulcatus complex, can serve as enzootic, or "cryptic", vectors; these rarely present a zoonotic risk because they rarely feed on humans (although they may feed on and infect companion animals such as dogs). The relative importance of enzootic vectors for contributing to the maintenance and diversity of LB spirochetes is undetermined.

\subsubsection{Host finding and host associations}

Both nest-associated (nidiculous or endophilic) and environment-associated (nonnidiculous or exophilic) species exist in the I. ricinus-persulcatus complex. The zoonotic vector species are exophilic and use an "ambush" strategy for contacting vertebrate hosts. They climb atop vegetation and wait for a passing vertebrate host to which to attach. At the simplest level, the likelihood of contact is a function of tick questing behavior, host abundance, and host behavior. Research on various I. ricinus-persulcatus complex ticks shows that they probably use several cues, including light, $\mathrm{CO}_{2}$, thermal, and host chemicals, to increase the probability of contacting hosts [255]. Genetic factors may also play a role.

The zoonotic vectors of LB are generalists and feed on a wide range of vertebrates, including mammals, birds, and lizards [59]. The spectrum of vertebrate host species, however, often varies among different life stages. Experiments suggest that abiotic factors influence questing height, thereby influencing host species use [222]. Immatures, which are more prone to desiccation, often host-seek low in the vegetation, where they can contact hosts of all sizes. Larvae feed on mammals of all sizes, ground-feeding birds and lizards. Adults, in contrast, host-seek higher in the vegetation and therefore parasitize mainly medium-sized and larger mammals [117]. Some tick species feed only on particular vertebrate species. For example, I. dentatus vectors $B$. burgdorferi in North America in enzootic cycles between birds and rabbits [270]. Even more specialized, I. uriae vectors $B$. garinii among pelagic colonial seabirds [188]. Indeed, population genetic studies of $I$. uriae in heterospecific seabird colonies suggest the sympatric existence of "host races", i.e. subpopulations of $I$. uriae that feed only on particular seabird species [158, 159].

\subsubsection{Feeding and development}

Using toothed chelicerae, ticks cut into the host's epithelial layers to create a feeding lesion, where they feed in a mire of fluid and blood [255]. Feeding is a gradual process during which periods of bloodsucking and salivation alternate. The completion of the blood meal requires several consecutive days, and its length varies with life stage, host species, and host immune status. For example, I. scapularis larvae feed for 3 to 5 days, nymphs for 4 to 5 days, and adult females for 7 days [66, 277]. Larvae and nymphs ingest approximately 10 to 20 times, and females 100 to 120 times, their unfed body weights [255]. Feeding is followed by a much longer off-host period during which engorged adult females lay a single batch of approximately a few thousand eggs, and engorged larvae and nymphs molt to the next life stage $[12,90]$. The developmental processes take weeks or months to complete and are influenced mainly by temperature-dependent and temperature-independent diapause [58, 223]. Life cycles in the I. ricinus-persulcatus complex range from 2 to 6 years, only a fleeting proportion of which is spent on a host [5].

\subsubsection{Dispersal and geographic range expansion}

No life stage of ticks in the I. ricinuspersulcatus complex crawls more than a few meters while questing $[35,61,89,255]$. Dispersal, both locally and more distantly, therefore depends entirely on the movement of vertebrate hosts and their behavior. For example, migratory birds and hosts with large home ranges (e.g. deer) should promote faster range expansion of ticks (and the pathogens they carry) than will hosts with smaller home ranges (e.g. mice) [146, 183].

\subsection{The vertebrate host}

A wide range of vertebrate species is parasitized by $I$. ricinus-persulcatus complex ticks (Fig. 1, Tab. I), but not all species are reservoir 
competent. A reservoir host must be able to: (i) host vector ticks; (ii) acquire the agent from infectious ticks; (iii) allow the agent to multiply and/or develop and persist in its body; and (iv) transmit the agent back to subsequently feeding vector ticks [115].

Decades of work in North America and Eurasia have revealed the importance of small mammals such as mice (e.g. genera Peromyscus, Apodemus and Neotoma), voles (e.g. genera Myodes [Clethrionomys] and Microtus), chipmunks (e.g. Tamias striatus and T. sibiricus), and shrews (e.g. genera Sorex, Neomys, and Blarina) for the enzootic transmission cycles of LB spirochetes [79, 127, 154, 271, 282]. Colonial seabirds and ground-foraging birds, such as pheasants (Phasianus colchicus), blackbirds (Turdus merula), European and American robins (Erithacus rubecula and T. migratorius) were soon recognized as being efficient reservoir hosts as well [46, 96, 121, 188, 231]. Medium-size mammals such as squirrels (Sciurus carolinensis, S. vulgaris, S. griseus), raccoons (Procyon lotor), opossums (Didelphis virginiana), and hares (Lepus europaeus and L. timidus) are involved to varying degrees in the enzootic cycles of LB spirochetes [46, 97, 107, 129, 141, 268]. Lizards rarely are reservoir hosts for pathogenic LB spirochetes in the USA, although certain species (e.g. Eumeces inexpectatus) may support enzootic cycles of LB spirochetes [43, 133, 266]. Green lizards (Lacerta viridis), sand lizards (L. agilis), and common wall lizards (Podarcis muralis) play a role in the enzootic cycles of $B$. lusitaniae in Europe $[2,147,156,234]$.

A number of vertebrate species are considered to be barriers for LB spirochetes. These hosts (e.g. deer, certain lizard species, and livestock in certain systems) are incompetent hosts [128, 180, 269], but feed vector ticks. These species thus can reduce, or "dilute" the proportion of infected ticks by diverting infected nymphs and naïve larvae away from reservoir hosts $[116,157]$. By providing blood meals to ticks, however, they nevertheless play important roles in increasing tick survivorship, the maintenance of the tick population (e.g. deer), and thus may facilitate the persistence of LB spirochetes (especially if non-systemic transmission may occur) [176]. Conversely, if ticks are host-limited, alternative hosts that have intermediate levels of reservoir competence may "rescue", or amplify, LB spirochetes [116]. Host species may also support different species and/or strains of LB spirochetes to varying degrees. Examples, and a likely mechanism responsible for the differences in reservoir competence, are discussed in Section 4.3.

\section{REPRODUCTIVE FITNESS $\left(\mathrm{R}_{\mathbf{0}}\right)$ AS A FRAMEWORK TO ASSESS MOLECULAR INTERACTIONS AMONG LB SPIROCHETES AND THEIR VECTOR AND VERTEBRATE HOSTS}

Given the continuing increase in the number of infected ticks in endemic foci as well as an expansion in the geographic range of infected vector ticks, what are the biological characteristics, or adaptations, that help make the LB system so successful?

Successful transmission requires complex interactions among the agent, the vector and vertebrate host. It can be difficult to see the relative importance of a particular interaction among the many. Thus, it is useful to construct simplified frameworks that represent the current understanding of the system. These frameworks allow researchers to organize their knowledge of the system and thereby identify gaps - from there, multiple competing hypotheses can be constructed, guiding future research directions.

Many authors have used the parasite triangle as a simplified framework $[179,218]$ : the triangle's vertices are represented by the pathogen, vector host, and vertebrate host (Fig. 1). The triangle illustrates the interdependency of the three organism types for the maintenance of the vector-borne agent and represents a community of interacting organisms - a "biocoenosis". The parasite community triangle is inseparable from the environment in which it sits, where it is under the constant influence of other biotic as well as abiotic factors. To synthesize the dynamic outcome of the parasite dynamics (i.e. enzootic, epizootic, or fadeout), the parasite triangle is linked with another - more quantitative - framework, $R_{0} . R_{0}$ is placed at the 
triangle's center to represent the summation of the triangle's processes in a given ecosystem.

$\mathrm{R}_{0}$ is the pathogen's reproductive number and is quantified in terms of numbers of infected hosts. It is unitless and is measured as the number of hosts that become infected as a result of the introduction of one infected host into a population of completely susceptible hosts [8]. $R_{0}$ therefore is a measure of the agent's reproductive fitness. If $\mathrm{R}_{0}=1$, one secondary case results from the primary case; the pathogen exactly replaces itself and is said to be endemic in the host population. If $\mathrm{R}_{0}>1$, many secondary cases result from the primary case, describing an epidemic. If $\mathrm{R}_{0}<1$, then the primary case fails to infect any susceptible individuals, and the pathogen goes extinct. In the case of LB spirochetes, one can ask, "given a population of susceptible hosts and an established population of vector ticks, how many future hosts will become infected with LB spirochetes upon the introduction of one infected host?"

Randolph and Craine [219] developed the first mathematical expression for $\mathrm{R}_{0}$ for a tickborne pathogen, which subsequently has been further developed analytically [99] (see Tab. II for further explanation and examples of factors influencing components of $\mathrm{R}_{0}$, Fig. 3 ). $\mathrm{R}_{0}$ is a summary of the entire parasite triangle conditional on the particular ecosystem, where processes within the parasite triangle are distilled into parameters within a mathematical expression. Therefore, $\mathrm{R}_{0}$ provides a framework to analyze the impact of traits and/or processes (e.g. molecular, organismal, ecological, environmental) in maintaining LB spirochetes in nature. For example, $\beta_{\mathrm{V}-\mathrm{T}}$ represents the transmission efficiency of LB spirochetes from an infected vertebrate to a naïve tick. In laboratory xenodiagnostic studies, $\beta_{\mathrm{V}-\mathrm{T}}$ can be estimated as the proportion of naïve larvae that become infected upon feeding on an infected host. Thus, the hypothesized importance of a particular phenotype of LB spirochetes for infection can be tested by quantifying $\beta_{\mathrm{V}-\mathrm{T}}$ and relating it to $\mathrm{R}_{0}$. For certain questions and depending on available data, the framework (i.e. parameters and the mathematical expression) can be modified to be more reductive or synthetical.
In the following section (Section 4), I highlight some of the biological traits and processes that enhance the transmission of LB spirochetes (i.e. favoring $R_{0} \geq 1$ ) in each of the major components of the parasite triangle. In addition, attention is paid to processes that facilitate interactions among LB spirochetes and their hosts, including vector-vertebrate interactions. Phenotypes (with a genetic basis) of LB spirochetes that favor $\mathrm{R}_{0} \geq 1$ would be considered adaptive. Table II lists the parameters of $R_{0}$ and examples of biological traits of the pathogen, vectors, hosts, as well as the interactions (detailed in the following sections) that would increase or decrease $\mathrm{R}_{0}^{2}$.

\section{INTERACTIONS AMONG LB SPIROCHETES AND THEIR VECTOR AND VERTEBRATE HOSTS}

To help categorize these interactions, I divide the transmission cycle of LB spirochetes into four key events: transmission between the vector and vertebrate hosts (occurring at least twice) and persistence between transmission events (occurring at least once in the vertebrate and at least once in the tick host). During the transmission events, all three organisms interact. The feeding lesion in the vertebrate host and the midgut of the tick represent the two theaters in which molecules from all three organisms perform. If transmission is successful, the drama continues for two of the three organisms; that is, the spirochete tries to persist in either the vertebrate or tick host until the next transmission event occurs. Here I focus on examples from the B. burgdorferi-I. scapularis system.

\subsection{Vector competency and LB spirochete transmission from vertebrate to tick $\left(\beta_{\mathrm{T}-\mathrm{V}} * \boldsymbol{\beta}_{\mathrm{T}-\mathrm{V}}\right)$}

When a susceptible tick parasitzes an infected vertebrate, what determines whether

\footnotetext{
${ }^{2}$ Phenotypes of the vector and vertebrate hosts that lead to $\mathrm{R}_{0} \geq 1$ would not be defined as adaptations of the pathogen, nor would they be considered adaptations of the vector or the host, unless they actually increased their fitness.
} 
Table II. A model (i.e. an hypothesis) of the sequence of steps for the determination of the susceptibility of a host to infection of a given LB spirochete strain/species (one component for determination of reservoir status) and transmission success from tick to vertebrate $\left(\beta_{\mathrm{T}-\mathrm{V}}\right)$. Susceptibility status is determined in the midgut of the feeding tick and is the outcome of interactions between the particular LB spirochete species and strain and the host species complement of the innate immune system. See text for more explanation of the tick to vertebrate transmission coefficient $\left(\beta_{\mathrm{T}-\mathrm{V}}\right)$ [219]. The relationship between spirochete load and $\beta_{\mathrm{T}-\mathrm{V}}$ may not be linear.

\begin{tabular}{|c|c|c|c|}
\hline \multirow[t]{2}{*}{ Step } & \multicolumn{3}{|c|}{$\begin{array}{l}\text { I. ricinus-persulcatus complex nymph infected } \\
\text { with a particular LB spirochete species or strain }\end{array}$} \\
\hline & Strain 1 & Strain 2 & Strain 3 \\
\hline $\begin{array}{l}\text { (1) Host blood enters midgut of infected nymphs; } \\
\text { spirochete population is mainly expressing OspA }\end{array}$ & Yes & Yes & Yes \\
\hline $\begin{array}{l}\text { (2) Can spirochetes bind host species-specific } \\
\text { regulatory proteins of the complement pathway and } \\
\text { avoid detection and destruction? (Outcome may vary } \\
\text { with host species.) }\end{array}$ & Yes & Somewhat & No \\
\hline (3) Spirochete status in the tick midgut & $\begin{array}{l}\text { Multiplies } \\
\text { profusely }\end{array}$ & $\begin{array}{l}\text { Multiples } \\
\text { moderately }\end{array}$ & Destroyed \\
\hline $\begin{array}{l}\text { (4) Spirochete population begins to upregulate OspC } \\
\text { and downregulate Osp A as they migrate from the tick } \\
\text { midgut through hemocoel to the salivary glands, and } \\
\text { then are transmitted to the host }\end{array}$ & Yes & Yes & None \\
\hline $\begin{array}{l}\left.\text { (5) Transmission efficiency ( } \beta_{\mathrm{T}-\mathrm{V}}\right) \text { to the host, } \\
\text { if all else equal. }\end{array}$ & $\begin{array}{l}\text { Higher } \\
\text { spirochete } \\
\text { inoculum } \\
\text { contributes to } \\
\text { greater } \beta_{\mathrm{T}-\mathrm{V}}\end{array}$ & $\begin{array}{l}\text { Moderate } \\
\text { spirochete } \\
\text { inoculum } \\
\text { contributes } \\
\text { to lower } \beta_{\mathrm{T}-\mathrm{V}}\end{array}$ & $\begin{array}{l}\text { No } \\
\text { transmission } \\
\left(\beta_{\mathrm{T}-\mathrm{V}}=0\right)\end{array}$ \\
\hline
\end{tabular}

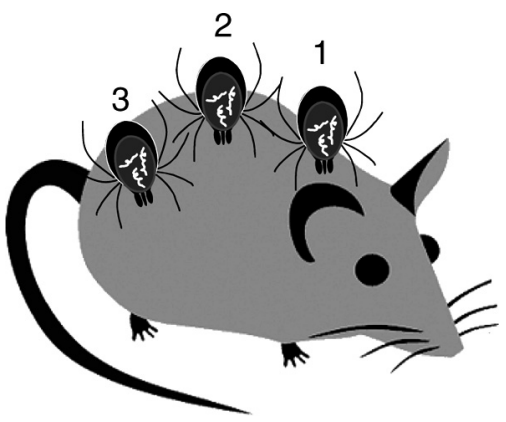

Figure 3. Three I. ricinus-persulcatus complex nymphs - infected with different LB spirochete species and/or strains - feeding on a reservoir host.

transmission will occur and the efficiency of transmission (i.e. what proportion of feeding vectors will become infected)? The extent to which LB spirochetes colonize a tick, reproduce, survive in the midgut through the molt, migrate to the salivary gland and then are transmitted to a susceptible vertebrate describes how "competent" a tick is for the spirochete. The degree of vector competency is a manifestation of many molecular interactions between the pathogen and tick. Here I consider two aspects of vector competency: (i) the ability of LB spirochetes to infect a tick $\left(\beta_{\mathrm{V}-\mathrm{T}}\right)$, and (ii) the ability of LB spirochetes to persist through the molt $\left(\beta_{\mathrm{T}-\mathrm{T}}\right)$. I discuss the third aspect of vector competency - the ability to transmit to a vertebrate host - in Section 4.3.

Together with ticks in the I. ricinus-persulcatus complex, other sympatric tick species have ample opportunities to become infected with LB spirochetes. I first summarize the studies 
that rule out the competency of two tick species that often share hosts with I. scapularis. I then turn to a brief discussion on tick innate immunity, which likely plays a large role in determining vector competence [257]. Finally I devote the rest of this section to highlighting particular B. burgdorferi-I. scapularis molecular interactions that illustrate our understanding of how B. burgdorferi is acquired and maintained through the molt. Any variation within the vector and LB spirochete populations for these and other traits can influence the relative fitness of various strains and therefore shape adaptations and evolution.

\subsubsection{Factors influencing $L B$ spirochete transmission from the vertebrate to the tick}

As with a reservoir host, a tick is vector competent if it is able to: (a) become infected with the agent when feeding upon a competent vertebrate; (b) maintain the agent through the molt; and (c) transmit the agent to a competent host during a subsequent blood meal [115]. Before exploring the traits that contribute to the high vector competency of I. ricinuspersulcatus complex vectors, it is worth examining recent research on mechanisms resulting in inefficient transmission in other tick species to improve understanding of mechanisms resulting in vector competence.

In the eastern USA, two human-biting ticks, Amblyomma americanum (the lonestar tick) and Dermacentor variabilis (the American dog tick), often overlap with I. scapularis in habitat, activity, and in vertebrate hostrange [23, 255]. Their prevalences of infection with LB spirochetes in the field are very low, however, and there is overwhelming laboratory evidence that both species are highly inefficient or incompetent vectors [155, 166, 201, 204, 239]. As will be explained below, different mechanisms prevent efficient vector competency in these two species.

A. americanum, the vector of the agents of human monocytic ehrlichiosis (Ehrlichia chaffeensis), canine ehrlichiosis (Ehrlichia ewingii), and possibly the unknown agent of southern tick-associated rash illness (STARI), commonly overlaps with I. scapularis in south- ern states and increasingly in northern states [3, $23,53,83,155,243$ ]. Field collections of questing and on-host $A$. americanum rarely have been infected with B. burgdorferi ${ }^{3}[86,273]$. Laboratory transmission experiments show that A. americanum larvae are: (i) infected with $B$. burgdorferi very inefficiently if at all $\left(\beta_{\mathrm{V}-\mathrm{T}} \sim 0\right)$ [155, 166, 201, 238, 239, 253]; (ii) highly inefficient at maintaining $B$. burgdorferi through the molt $\left(\beta_{\mathrm{T}-\mathrm{T}} \sim 0\right)$ ) [155, 201, 239]; and (iii) incapable of transmitting the spirochete to susceptible hosts $\left(\beta_{\mathrm{T}-\mathrm{V}}=0\right)$ [238]. Ledin et al. [132] found that $A$. americanum saliva effectively kills $B$. burgdorferi - fewer than $13 \%$ of spirochetes that were exposed to saliva for $48 \mathrm{~h}$ remained alive. Thus, most spirochetes in the feeding lesion of a host do not survive to infect $A$. americanum. The borreliacidal factor(s) has (have) not yet been identified, but may be related to observed differences in the sialomes (i.e. set of mRNAs and proteins found in salivary glands) of $A$. americanum and I. scapularis [281].

D. variabilis, the vector for the agent of Rocky Mountain spotted fever (Rickettsia rickettsii), overlaps much of I. scapularis' range $[23,53]$. In contrast to $A$. americanum, engorged $D$. variabilis removed from wild hosts and dogs have been found to harbor B. burgdorferi spirochetes $\left(\beta_{\mathrm{V}-\mathrm{T}}>0\right)[4,94]$, and a low percentage of questing adults have been found to be infected with viable B. burgdorferi spirochetes, indicating that ingested spirochetes can be maintained transstadially, albeit inefficiently $\left(\beta_{\mathrm{T}-\mathrm{T}}>0\right)[204,283]$. Nevertheless, laboratory experiments have shown that $D$. variabilis nymphs that previously had fed as larvae on $B$. burgdorferiinfected hosts subsequently are unable to infect susceptible hosts $\left(\beta_{\mathrm{T}-\mathrm{V}}=0\right)[155,166,201$, 239]. The combination of field and laboratory

\footnotetext{
${ }^{3}$ While a few older studies do report finding $B$. burgdorferi-infected field $A$. americanum, it is possible that the ticks were infected with B. lonestari, a relapsing fever spirochete discovered in the late 1990s that would not have been distinguishable from $B$. burgdorferi in the diagnostic assays used by those studies [15].
} 
data strongly indicates that $D$. variabilis is ecologically unimportant as a vector.

Laboratory studies suggest that $D$. variabilis kills spirochetes after ingestion. Johns et al. $[110,111]$ showed that when B. burgdorferi organisms were injected into the hemocoel of D. variabilis, there was an increase in hemocyte count and subsequent lysis and reduction of live spirochetes. These authors also identified an antimicrobial peptide, a defensin with borreliacidal properties, whose production is increased in response to $B$. burgdorferi challenge, leading to rapid spirochete lysis [112]. While I. scapularis hemolymph also exhibits moderate borreliacidal activity, killing seems to depend on cell-mediated processes rather than antimicrobial peptides. Johns et al. hypothesize that the cell-mediated process is much slower in I. scapularis, allowing spirochetes time to disseminate to tissues such as the salivary glands, where they can evade the tick's immune response. Furthermore, although a defensin also has been found in I. scapularis, it does not appear to have borreliacidal activity [108]. These data explain in part how $D$. variabilis can acquire $B$. burgdorferi from an infected host and maintain some spirochetes through the molt, but then cannot transmit them to reservoir hosts [253].

These studies implicate the role of innate immunity, which is much less understood for ticks than for insects, in determining the degree of vector competency [257]. Among B. burgdorferi vectors, competency levels vary. Compared to northern populations of I. scapularis, infection prevalence of questing ticks is generally lower in I. pacificus populations [60], and much lower in southern populations of $I$. scapularis $[42,187]$. While ecological conditions seem to limit the enzootic dynamics of $B$. burgdorferi in those areas (see Section 5), genetic differences in innate immunity may also contribute to the observed variation. Some early laboratory experiments [203] showed that I. pacificus is less efficient at acquiring and maintaining B. burgdorferi spirochetes compared with northern and southern populations of I. scapularis [201], which were comparable. A more comprehensive comparison of similar studies is not possible because different researchers used different methods to infect hosts, laboratory hosts, and spirochete strains. Motivated to design prophylactic therapeutics and vaccines, researchers surely will uncover more innate immune mechanisms influencing vector competence of $I$. ricinus-persulcatus complex vectors.

\subsubsection{Successful colonization of vectors by $\mathrm{LB}$ spirochetes}

Despite exhibiting some borreliacidal activity $[111,132]$, I. scapularis is a highly efficient vector for $B$. burgdorferi. Research in the last decade has identified several proteins expressed by $B$. burgdorferi in the tick environment that seem to be important for pathogen acquisition and persistence in the tick; these have been described in detail in several previous reviews (e.g. [10, 64, 104, 191]). Here I highlight a well-characterized molecular interaction at the B. burgdorferi-I. scapularis interface that results in a high degree of vector competence.

Several outer surface proteins have been shown to be necessary for successful infectivity of $I$. scapularis by B. burgdorferi $\left(\beta_{\mathrm{V}-\mathrm{T}}\right)$. Both outer surface proteins (Osp) A and B are preferentially expressed by $B$. burgdorferi when colonizing the tick, and then are generally down-regulated during tick feeding and when in the vertebrate host $[30,50,52]$. OspA has been found to bind to a tick midgut receptor, TROSPA [192]. Experiments using antibodies against OspA as well as RNAi manipulations to inhibit expression of OspA by the spirochete or expression of TROSPA by the tick show that OspA is critical for spirochete colonization [49, 190, 192, 299]. OspA has also been found to stick to itself, and thereby may further facilitate spirochete-spirochete adherence and interaction [190]. OspB, which shares a promoter with OspA [106], also seems necessary for B. burgdorferi adherence to the tick midgut, although the putative midgut receptor has not been identified [63, 174, 299]. OspA and B probably help $B$. burgdorferi to remain attached to the midgut, rather than being actively removed or destroyed by the tick, during feeding and/or molting. Thus, along with the lack of a strong innuate immune response against $B$. burgdorferi, expression of the TROSPA receptor contributes 
to the vector competence of $I$. scapularis. Within the $\mathrm{R}_{0}$ framework, OspA and $\mathrm{B}$ (and other proteins like BB0365, [194]) seem critical to increasing both the transmission coefficient from vertebrate to tick $\left(\beta_{\mathrm{V}-\mathrm{T}}\right)$ and the transstadial transmission coefficient $\left(\beta_{\mathrm{T}-\mathrm{T}}\right)$, and thus are considered to be adaptive traits.

With the recent availability of several LB spirochete genome sequences, high throughput molecular techniques, and sophisticated molecular manipulations in combination with transmission experiments, researchers will continue to characterize spirochete proteins and their phenotypes. Some, like OspA, will be critical for successful LB spirochete infection, persistence, and transmission; others may not be essential [177]. For example, OspD expression is highly correlated with $B$. burgdorferi replication, which occurs while the tick is feeding and digesting its meal, but ceases after the molt [263]. OspD-mutants have lower colonization rates in the tick midgut, which results in lower spirochete burdens but not in lowered transmission to susceptible hosts [138]. This finding of a partial effect of a protein on acquisition or maintenance of B. burgdorferi implies that multiple genes and gene products are acting in a compensatory manner [138, 194, 297], although it may also be that researchers have not found the context in which a particular protein plays a critical role.

\subsection{LB spirochete persistence in the tick $\left(\beta_{\mathrm{T}-\mathrm{T}}\right)$}

Once $B$. burgdorferi successfully colonizes the midgut of I. scapularis, it must survive the midgut environment during the blood meal as well as through the molt and then persist in the midgut until the next blood meal. During the larval blood meal and just after attachment, the spirochete population within the midgut continues to increase [253]. The population then decreases either before or after the molt $[32,202]$. This decrease can represent an inability to remain anchored to the midgut epithelium and/or to a lack of necessary resources such as $\mathrm{N}$-acetyl-glucosamine [32, 202]. The decrease in spirochetes in flat nymphs represents a bottleneck in population size (and perhaps diversity as well). Furthermore, the spirochete burden in a flat $I$. scapularis nymph seems to be a critical component for infection, as below a certain threshold, infected nymphs may not successfully infect vertebrate hosts [49].

Surviving spirochetes must persist in the midgut until the tick contacts another vertebrate host. In the northeastern USA, approximately 9 to 10 months elapse between the peak larval and nymphal host-seeking periods, and approximately 6 months elapse between the peak nymphal and adult host-seeking periods. $\mathrm{Bb} 0690$ is a $B$. burgdorferi protein that is a homolog of Dps, a DNA-binding protein known to protect DNA against damage during starvation or oxidative stress in Escherichia coli and other bacteria. Dps provides a critical role in preserving the integrity of $B$. burgdorferi DNA in flat ticks between blood meals. Li et al. [139] infected I. scapularis larvae with Dps-deficient B. burgdorferi, allowed them to molt, and then challenged naive mice with nymphs of two ages - 3 weeks and 4 months post-ecdysis. For the younger nymphs, spirochete burdens in engorging ticks were similar to those in control, infected nymphs, and transmission to mice was $100 \%$. For the older nymphs, however, spirochete burdens plummeted and no transmission to mice occurred. Furthermore, they show that Dps expression is highest in questing ticks and lowest in feeding and engorged ticks. Consequently, they hypothesize that BB0690 is crucial for B. burgdorferi survivorship, and thus adaptive, during the inter-blood meal period, but not for transmission per se. Future studies likely will reveal other proteins that aid LB spirochete persistence during off-host periods of the tick's lifecycle.

\subsection{LB spirochete transmission to the vertebrate host $\left(\beta_{\mathrm{T}-\mathrm{V}}\right)$}

Transmission of B. burgdorferi by nymphal I. scapularis generally requires 40 to $48 \mathrm{~h}$, during which the spirochete replication in the engorging tick is followed by migration through the hemocoel and invasion of the salivary glands [103, 200] (although transmission of LB spirochetes by nymphal $I$. ricinus may occur sooner, illustrating variation among different LB systems [114]). During this time, the drama that heavily influences spirochete transmission 
$\left(\beta_{\mathrm{T}-\mathrm{V}}\right)$ occurs in the tick midgut, where the spirochetes and host factors in the blood meal interact. The quantitative nature of these interactions likely varies with the particular combination of the LB spirochete species/strain and the host species [51, 98]. While this review highlights how certain salivary molecules affect both the transmission and acquisition of LB spirochetes at the feeding lesion, and how antimicrobial peptides interactions with spirochetal factors in the midgut can influence these transmission coefficients, a more complete understanding of threeway interactions among spirochetes, the tick and the host would be a fascinating area for future research.

The many different vertebrate host species provide a myriad of ecological niches in which LB spirochetes can exist. As with other organisms, LB spirochete species vary in their degree of host specialization: some genotypes (both among and within species) are niche specialists adapted to phylogenetically closely related vertebrates (e.g. mammals or birds), while others are generalists that can exploit more than one such niche [122, 123].

The quality of a vertebrate species as a niche for LB spirochetes seems to be largely mediated by species-specific innate immunity, which comes into play as soon as the spirochetes contact complement in the host blood meal [123]. At the beginning of infection, before any pathogen-specific antibodies are made, the invading spirochetes are attacked by the non-specific alternative complement pathway. Deposition of complement components such as $\mathrm{C} 3 \mathrm{~b}$ on surfaces of invading pathogens results in opsonization and direct killing by the formation of membrane attack complexes. C3b deposition, in the alternative pathway, occurs spontaneously and indiscriminately on host and pathogen surfaces that are in contact with blood. To avoid the destruction of host cells, a number of regulatory proteins regulate $\mathrm{C} 3 \mathrm{~b}$ deposition.

Interestingly, LB spirochetes express several complement regulator-acquiring surface proteins - such as BbCRASP-1, BbCRASP-2, BbCRASP-3, OspE, and other Erp proteins (of which each spirochete may possess multiple forms). These proteins can bind regulatory proteins of the alternative complement pathway, such as factor $\mathrm{H}$ and factor $\mathrm{H}$-like protein 1 (FHL-1) [1, 100, 118, 119, 260, 284]. Once coated with these host regulatory proteins, spirochetes avoid recognition and eradication by complement. Thus it appears that the host range for LB spirochetes is determined by their sensitivities to complement of particular host species [260], and furthermore, the ability to bind factor $\mathrm{H}$ and FHL-1 appears to depend on the genotype of LB spirochetes [123].

Because complement-mediated selection operates directly in the midgut of feeding ticks, spirochetes are destroyed directly in the ticks, prior to transmission to the host. The interaction between spirochetes with complement therefore has a major impact on LB spirochete transmission dynamics and $\mathrm{R}_{0}$ in nature. For example, it is hypothesized that the paucity of infected I. pacificus nymphs is due to the sensitivity of B. burgdorferi to complement of western fence lizard (Sceloporus occidentalis), and because I. pacificus immatures tend to feed on lizards more than sympatric rodents [128].

\section{Antigenic switching of $L B$ spirochetes in the tick and interactions with tick salivary pro- teins enable early infection of reservoir hosts $\left(\beta_{T-V}\right)$.}

When an infected host-seeking nymph contacts a host and begins to feed, an increase in $B$. burgdorferi replication in the midgut is accompanied by differential regulation of various genes. One oft-cited shift is the downregulation of $o s p A$ and up-regulation of $o s p C$ in spirochetes in the midgut $[148,186,245$, 246]. While not all spirochetes undergo this transformation, there is proportionately lower OspC expression by spirochetes in the midgut of flat (i.e. unfed) ticks, and there is little OspA expression by spirochetes in the salivary glands or feeding lesion. This shift in the expression of this pair of genes has become a paradigm for the differential expression of suites of genes associated with different steps in the natural transmission cycle of LB spirochetes between the tick and vertebrate hosts. In the case of OspC, an abundantly expressed outersurface protein, this shift reduces the effect of the host adaptive immune response to eliminate spirochetes in the tick midgut. Because spirochetes 
downregulate OspC expression in the tick midgut, host anti-OspC antibodies ingested by an infected tick cannot prevent transmission from the tick to the vertebrate.

OspC expression is necessary for early infection of the host during natural tick-mammal transmission [92, 262], but can have variable expression in later infection [101]. OspC aids in evading the host immune system during early infection as well as during dissemination, but a precise understanding of the mechanisms remains elusive [215]. Although OspC was found to bind to the tick salivary gland [193], ospC-mutants still can migrate to the salivary glands [92], but are unable to establish early infection in mammalian hosts [275]. Subsequently, it was discovered that OspC binds to the salivary protein Salp15 [216], which previously had been found to inhibit CD4+ T cell activation [9, 75]. Thus, binding to Salp15 may protect $B$. burgdorferi from antibodymediated killing in the host and thereby facilitate successful early infection. Within the $\mathrm{R}_{0}$ framework, the interaction between OspC and Salp15 may increase $\beta_{\mathrm{T}-\mathrm{V}}$ (and even $\beta_{\mathrm{V}-\mathrm{T}}$ if nonsystemic transmission occurs) and thus be adaptive for $B$. burgdorferi. RNAi silencing of Salp15, however, does not completely inhibit spirochete transmission [216]. Thus there may be other proteins to which OspC binds or mechanisms by which OspC aids in the transmission of B. burgdorferi. Similarly, Xu et al. [297] showed that other lipoproteins (OspA, VlsE, OspE, and $\mathrm{DbpA}$ ) can compensate for the lack of OspC in aiding both early infection and dissemination prior to the initiation of the adaptive immune response.

\subsection{Persistence of infectious LB spirochetes in the vertebrate host $(\mathbf{r})$}

Once LB spirochetes gain access to the vertebrate host, they need to survive until they are transmitted back to competent ticks. This effectively means that the recovery (r) and mortality (h) rates of the vertebrate must be low relative to the contact rate with another tick. Here "recovery" specifically refers to the host's loss of ability to transmit spirochetes to another tick, i.e. due to immunity. The B. burgdorferi gen- ome does not seem to contain known virulence genes $[37,73,276]$, so it appears at this time that successful infectivity of and persistence within a mammal host depend primarily on evading the host's immune system, rather than exploiting the host tissues for reproduction and growth $[85,216,276]$. Within the vertebrate host, the spirochetes are localized first in skin near the feeding lesion (giving rise to the diagnostic erythema migrans or "bull's eye" rash), then travel through the bloodstream, and finally disseminate to skin and other varied organs such as joints, heart, and bladder. It is hypothesized that spirocehetes persist in the extracellular matrix of such organs until transmission to another feeding tick can be attempted (Fig. 4) [236]. While this sequence of events has been thus documented, the relative importance of the roles of hematogenous and extravascular dissemination for eventual transmission of spirochetes to subsequently feeding ticks is unknown [124].

\subsubsection{Early infection: evading the host's immune system}

The mechanisms underlying evasion by LB spirochetes from host defenses during early infection depend critically on functional proteins being present in adequate quantities within a crucial time window. Among these proteins is OspC, which was previously discussed in the context of "preparing" spirochetes in ticks for transmission to the vertebrate and which is critical for early infection of mammalian hosts [92, 215, 262]. Also, as mentioned earlier, even prior to infection of the host, CRASP and Erp proteins play an important role in helping LB spirochetes evade host complement-mediated killing.

\subsubsection{Spirochetemia}

To continue evading the host immune system, LB spirochetes eventually down-regulate OspC and introduce new antigenic actors. One of the mechanisms that help LB spirochetes cope with host adaptive immunity is antigenic variation of an outer membrane lipoprotein, expressed by the variable major 

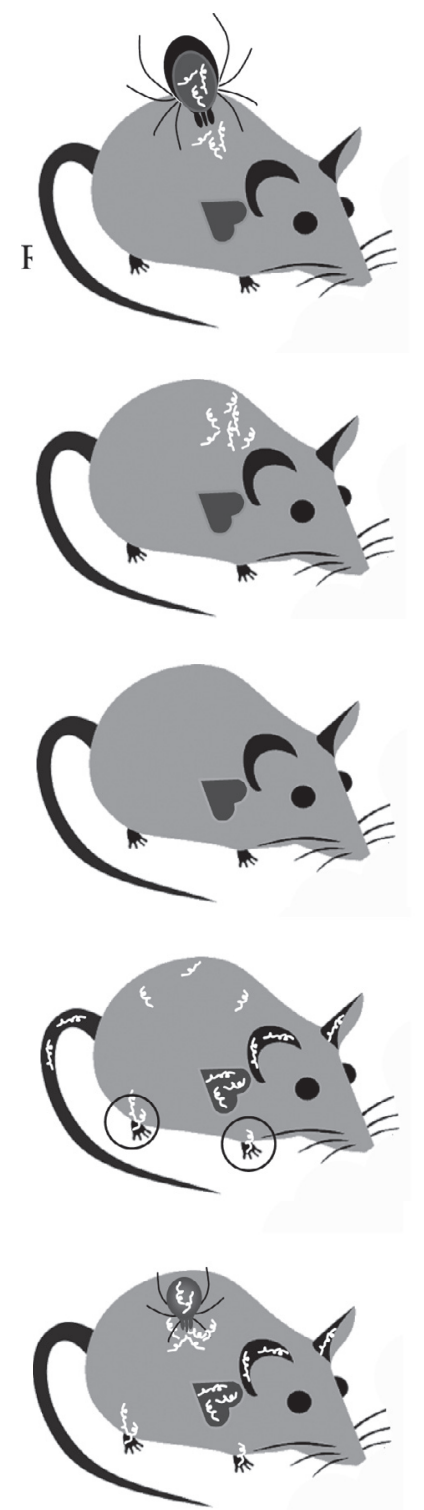

(1) Transmission of spirochetes into the feeding lesion in the dermis. Tick salivary proteins and expression of OspC by the spirochete help suppress and evade the immune system.

(2) Early infection. Spirochetes disseminate locally in the dermis; expression of CRASPs and Erp proteins help to evade the innate immune system.

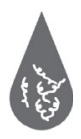

(3) Dissemination. Spirochetes enter the bloodstream, exhibiting antigenic variation (e.g. vlsE protein) evading the adaptive immune response. Spirochetemia is brief, on the order of 10-14 days in mice.

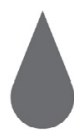

(4) Late infection. Spirochetes exit the bloodstream and invade tissues, continuing to evade the adaptive immune system, potentially protected in immune privileged sites, such as the extracellular matrix.

(5) Transmission to ticks. Spirochetes migrate to the feeding lesion of subsequently feeding ticks, where they may be transmitted. (What is the fate of spirochete populations that colonize internal organs?)

Figure 4. Infection and persistence of spirochetes in the reservoir host and transmission to subsequently feeding ticks. LB spirochete species and strains may vary in duration of persistence and infectivity $(\mathrm{r})$ in a given host species [51, 98], and therefore the host-to-tick transmission coefficient $\left(\beta_{\mathrm{V}-\mathrm{T}}\right)$ [219]. See text for further explanation.

protein-like gene $(v l s E)$. The $v l s$ system consists of a $v l s$ expression site ( $v l s E$ ) encoding a surface-exposed lipoprotein and 15 unexpressed silent $v l s$ cassettes. The vlsE protein consists of six highly variable regions interspersed among invariable regions. In mice, segments from variable regions of the silent cassettes randomly recombine into the $v l s E$ gene as soon as 
four days post-infection [301]. During the infection LB spirochetes establish a large repertoire of VlsE variants that alter its antigenicity, despite the robust antibody response to conserved segments. Variation in VlsE was not observed in vitro nor in ticks, suggesting that recombination is promoted by unidentified host factors [178], such as ones participating in the host inflammatory response [160].

\subsubsection{Disseminated infection and persistence}

After the host adaptive immune response becomes effective (on the order of 7 to 14 days in mice and humans, but dependent on the strain or genotype [55, 286]), spirochetes disappear from the bloodstream but have disseminated to internal organs, synovial fluid, and collagenous tissue in the skin, joints, and tendons [101]. Spirochetes may find protection in these sites from the host immune system. For instance, while passive transfer of immune sera can prevent infection of naïve hosts with $B$. burgdorferi, it cannot cure previously infected hosts [16]. Spirochete populations found in different sites $[17,195]$, however, may vary in susceptibility to antibodies, suggesting either tissue-dependent binding by spirochetes or tissue-dependent efficacy of antibodies. Furthermore, although antibiotics are very effective at clearing infections, PCR assays have detected residual spirochetes in some mice, dogs, and humans after treatment (see later in this Section 4.4) [25, 212, 264, 265].

The extracellular matrix (ECM) may provide an immune-privileged environment for spirochetes [33, 102]. Several B. burgdorferi molecules have been identified that help spirochetes attach to ECM elements such as proteoglycan decorin, glycosaminoglycans, fibronectin, and host cells ([33], and references therein). Empirical manipulations, such as antibodies against Dbp A and B, can reduce the infectivity of $B$. burgdorferi for various tissues and spirochete loads, but as with other processes, $B$. burgdorferi seems to have redundant adhesive interactions in place to ensure persistence in the ECM [17, 33, 249]. For example, Pal et al. [195] showed that BmpA and $\mathrm{B}$ are two spirochete lipoproteins that are upregulated by spirochetes in joint tissue, required for colonization of joint tissues, and contribute to arthritis in mice.

LB spirochetes' interactions with certain other host cells may further help evade the immune system either intracellularly or extracellulary. B. burgdorferi has been found intracellularly in human endothelial cells, macrophages, and cardiac myocytes [144, 162, 189]. Extracellularly, B. burgdorferi can bind to the invaginations of cell membranes of fibrocytes, thereby avoiding phagocytosis as well as antibodies and antibiotics [77, 88, 175]. Future studies undoubtedly will uncover other spirochete-host cell interactions, as well as their relative importance for $B$. burgdorferi dissemination and persistence in the vertebrate host.

\subsubsection{Prelude to transmission to ticks: migration of spirochetes to feeding lesion}

The successful migration of spirochetes from within the vertebrate host into the feeding tick is not well understood. Presumably aggregation of spirochetes around a tick feeding lesion results in increased transmission efficiency $\left(\beta_{\mathrm{V}-\mathrm{T}}\right)$ of the pathogen from host to ticks. Chemotaxis probably helps direct LB spirochetes towards feeding ticks $[38,142]$. Salivary gland extracts of fed ticks act in vitro as an attractant for B. burgdorferi [250].

Narasimhan et al. [171] provide an in vivo example illustrating how the spirochetes might use vector molecules to increase the success of transmission from host to ticks. The tick salivary protein Salp25D helps detoxify reactive oxygen species produced by neutrophils at the feeding lesion, thereby increasing B. burgdorferi survivorship as spirochetes run the gauntlet from the mammal to the tick midgut. When Salp25D is silenced and/or when the mouse host is immunized against Salp25D, significantly fewer spirochetes colonize midgets of feeding larvae compared with controls (reducing $\beta_{\mathrm{V}-\mathrm{T}}$ ) [171]. The authors did not report the subsequent transstadial transmission coefficient $\left(\beta_{\mathrm{T}-\mathrm{T}}\right)$, but if the proportion of infected molted nymphal ticks were lower than that of controls, it would further confirm a positive effect of Salp25D on spirochete fitness. 
An emerging paradigm is the potential role of hormone molecules that can signal the presence of feeding ticks to the spirochete. Scheckelhoff et al. have found that binding by $B$. burgdorferi to the human catecholamines epinephrine and norepinephrine results in increased expression of OspA (predominantly expressed in ticks and not vertebrates) [242]. Furthermore, when researchers administered an inhibitor of catecholamine receptors to infected mice, a lower proportion of feeding ticks became infected compared to ticks feeding on control infected mice. Catecholamines also can be found in tick saliva [242]. Thus, whether it is a signal of stress by the host due to infestation with a tick, or a salivary protein signaling the presence of a feeding tick, hormones may aid in transmission of spirochetes to vector ticks.

It would appear that spirochetes persisting in the skin have a greater probability of being transmitted to ticks, given the average duration of tick engorgement (e.g. 3 to 5 days for larval I. scapularis on mice). Thus interactions that allow spirochetes to evade the host immune system, recolonize skin, and navigate through the ECM can be inferred to be adaptive (e.g. [164]). What are the fates, however, of spirochetes that persist in the ECM of internal organs and tissues (e.g. joints, ligaments, and synovial fluid)? Evolutionarily, these populations of spirochetes will be dead ends if they are unable to migrate to tick-feeding sites and infect hosts. An intriguing, recent study suggests that spirochetes persisting in internal organs (e.g. heartbase and tibiotarsus) of laboratory $\mathrm{C} 3 \mathrm{H}$ mice are capable of transmission to xenodiagnostic larvae [102]. In that study $\mathrm{C} 3 \mathrm{H}$ mice had been previously infected by needle inoculation and then treated with antibiotics 1 or 3 months post-infection. Spirochetes were not detectable or culturable at any sites, including three different skin sites, and were PCR-positive only in internal organs. Xenodiagnostic larvae were allowed to molt, and as nymphal ticks, they were able to infect $\mathrm{C} 3 \mathrm{H}$ scid (i.e. severe combined immunodeficiency) mice. Prior to this study, it had been thought that these spirochetes, which were detected in joints and various other organs by PCR, were unlikely to be infectious because they were unculturable [25]. The inference from this recent study is that the transmitted spirochetes were not residents of skin, but from persistent populations in internal organs. Further understanding the impact of these transmittable, but unculturable, spirochetes on natural transmission cycles or disease requires investigating whether these spirochetes can infect immune competent mice.

If these persistent spirochetes are rarely transmitted to immune competent hosts and therefore rarely contribute to the reproductive population of spirochetes, the "adaptive" value of the interactions is questionable (although potentially important for understanding the etiology of disease). Furthermore, an hypothesis would be that these interactions are the byproducts of adaptations that help B. burgdorferi populations in the skin become transmitted to a tick, but which cause some subpopulations of spirochetes to locate to "dead-end" tissues.

\subsection{Vector-host interactions and effects on LB spirochete transmission}

Broadly speaking, vertebrate host-tick interactions that influence LB spirochete populations include the processes whereby ticks (1) contact hosts and (2) successfully obtaining replete blood meals from hosts. Both processes influence tick fitness and tick population structure such that adaptations for increasing tick feeding success should benefit both tick and spirochete populations, with the caveat that some vertebrate host species (e.g. white-tailed deer) are suitable hosts for ticks, but incompetent, or barrier, hosts for LB spirochetes. Here I will discuss the on-host interactions between ticks and hosts, focusing at the sub-tick and sub-host level and reserving discussion of the ecological factors for a later section (Section 5). These tick-host interactions may enhance the transmission coefficients (both $\beta_{\mathrm{T}-\mathrm{V}}$ and $\beta_{\mathrm{V}-\mathrm{T}}$ ).

There have been many review articles [29, 288] written about tick-host interactions, as well as tick-host-pathogen interactions, as researchers have come to recognize the importance of the former on pathogen transmission [179, $218,289,290]$. While researchers noted decades ago the benefits of tick salivary gland 
extracts on tick feeding success and pathogen transmission [18], advances in modern molecular, genomic and proteonomic tools have increased our ability to "see", characterize, and conduct experiments to infer the functions of specific salivary proteins. It is beyond the scope of this review to catalog all the different salivary genes, transcripts, and proteins that may play an important role in enhancing LB spirochete transmission either directly or indirectly. Here, I highlight a few of the interactions to illustrate how they may influence survivorship, transmission, and fitness of LB spirochetes and refer the readers to the several excellent recent reviews and articles on the topic [29, 104, 179, 229]. An important point that previous authors have made is that just as the vertebrate host's responses have built-in redundancy, so do the counteractive responses of the tick [226, 229].

\subsubsection{The pharmacopeia of the feeding lesion: enhanced feeding success}

Just as a tick has gross morphological adaptations to help it attach to a host, it also secretes microscale adaptations in the form of salivary proteins to secure a replete blood meal. The invasive injury to the integrity of the host's tissues and blood vessels at the feeding lesion alerts the host's repair and immune systems and triggers a cascade of processes that threaten to terminate feeding and thereby reduce the tick's survivorship and the fitness. All else equal, ticks possessing salivary proteins that can help them evade, mitigate, or modulate host responses will feed more successfully and produce more progeny. Thus, there is strong evolutionary pressure for the selection of "countermeasure" salivary proteins.

To maintain hemostasis, the host responds by vasoconstriction, platelet aggregation, and coagulation. Maritz-Olivier et al. [151] provide a recent review on tick anti-hemostatics and highlight how these factors may be potential targets for anti-tick vaccines and therapeutics. Ribeiro et al. [226] first identified the presence of platelet aggregation inhibition factors, such as an apyrase, and noted an abundance of prostaglandin $\left(\mathrm{PGE}_{2}\right)$, a vasodilating effector, in I. scapularis saliva. Two proteins that I. scapularis produces that prevent coagulation are ixolaris and penthalaris, which are both tissue factor pathway inhibitors [69, 71, 161]. Additionally, ixolaris recently was found to have anti-thrombotic properties [172]. Homologous transcripts for both of these proteins have been found in the sialome of I. pacificus as well [72]. Another anticoagulant, Salp14, inhibits the intrinsic pathway of coagulation [169, 170]. Finally, MP1, a fibrin(ogen)olytic agent that aids in dissolving bloodclots, has been identified [70]. Inhibition of bloodclotting may be beneficial within the tick midgut as well as in the feeding lesion.

Processes in the hemostasis response also lead to and/or interact with inflammatory processes, resulting in redness, heat, swelling, and ultimately, pain. Pain and itchiness may evoke grooming behavior, unless the tick is able to reduce the inflammation response. I. scapularis possesses a salivary metallo dipeptidyl carboxypeptidase [226, 228] that inactivates bradykinin, a molecule in the hemostasis pathway that also can elicit a pain response. I. scapularis also has a number of proteins that inhibit the alternative complement pathway and prevent the formation of anaphylatoxins, thereby reducing both inflammatory and immunological responses. In particular, Isac [279, 281] belongs to a family of anti-complement proteins [230] that have been shown experimentally to enable successful feeding [253].

Given the relatively long duration of feeding required to obtain a replete blood meal, a tick is highly susceptible to host immunological responses, particularly if the host previously had been fed upon by other ticks. Acquired immunological resistance to ticks manifests as a longer feeding duration to complete engorgement, lower engorgement rate, and decreased egg production (for adult females), and therefore, lower survivorship probabilities and/or fitness for the tick. Both the adaptive immune response (i.e. antibodies to salivary proteins) and the alternative pathway of complement activation are involved with acquired resistance [29].

I. scapularis possesses a diversity of salivary proteins and mechanisms to combat the host 
immune system, and our knowledge of these proteins continues to grow. Ribeiro et al. [226] demonstrated that I. scapularis saliva inhibited $\mathrm{T}$ cell proliferation, and researchers subsequently identified an IL-2 binding protein $[80,280]$ as the putative mechanism. Inactivation of $\mathrm{T}$ cell proliferation affects both the humoral and cellular immune responses. Salp15 inhibits CD4+ T cells activation as well as IL-2 production [9], which can down-regulate Th1 response and therefore macrophage activity. Subsequently, it has been learned that Salp15 also inhibits pro-inflammatory cytokines (IL$12 \mathrm{p} 70$, IL-6, and TNF-b) production by binding to a lectin, DC-SIGN, on dendritic cells [105]. This finding is significant as dendritic cells, which are already present and common at feeding lesions, play a central role in initiating these adaptive immune responses.

\subsubsection{The pharmocopeia of the feeding lesion: enhanced LB spirochete transmission}

Acquired resistance to feeding ticks can reduce pathogen transmission in both directions (i.e. both $\beta_{\mathrm{T}-\mathrm{V}}$ and $\beta_{\mathrm{V}-\mathrm{T}}$ ). Nazario et al. [173] showed how repeated infestations of guinea pigs with uninfected I. scapularis ticks prevented transmission of $B$. burgdorferi from infected ticks feeding subsequently. This effect is not surprising - due to a lack of co-evolution, the tick and the unnatural host species have not fine-tuned their countermeasures. In nature, however, there exists a spectrum of interactions among various host species and ticks, representing different genetic, ecological and coevolutionary histories. Some hosts, like P. leucopus mice in North America, can produce consistently infected ticks among multiple feedings [48] and even enhanced transmission in the case of A. flavicollis mice in Europe [78]. Other natural hosts, like unnatural laboratory hosts, however, develop acquired resistance, leading to reduced pathogen transmission. Davidar et al. [48] show that meadow voles (Microtus pennsylvaticus), although reservoir competent for $B$. burgdorferi, mount anti-tick immune responses to repeated tick infestations, resulting in reduced transmission. Acquired resistance in meadow voles resulted in larvae with lower engorgement weights and lower molting success compared to larvae that fed on previously infested white-footed mice. Similar trends were found between European voles and mice [54, $78,120]$.

Other experiments with unnatural hosts have revealed the impacts that tick saliva can exert on pathogen transmission. Ribeiro et al. [227] showed that I. scapularis saliva could inhibit rat neutrophil function, and in the process also prevent $B$. burgdorferi from phagocytosis. Zeidner et al. [300] demonstrated in laboratory mice that inhibition of host cytokines by I. scapularis saliva is critical for $B$. burgdorferi transmission. Intuitively it makes sense that the anti-haemostatic, anti-inflammatory, and the immunosuppressive properties of tick saliva would also promote pathogen transmission. As mentioned previously, Salp25D is an antioxidant that critically enables $B$. burgdorferi acquisition by detoxifying the reactive oxygen species that is part of the host's immune response to tick feeding [171]. It is in this regard that Wikel [289] writes, "the tick is clearly not just a crawling hypodermic needle and syringe with regard to the transmission of tick-borne pathogens".

Nuttall and Labuda [179] have coined the term "saliva-activated transmission" (SAT) to describe the enhanced transmission of pathogens at the tick feeding site. SAT can help facilitate transmission of pathogens from an infected feeding tick to another uninfected tick even in a host whose serum normally may neutralize LB spirochetes (i.e. is considered an incompetent host) or that has mounted antibodies against the pathogen. Non-systematic, or co-feeding, transmission via SAT has been demonstrated as the main route of transmission by which tick-borne encephalitis virus (vectored by I. ricinus and I. persulcatus) is maintained in nature [220, 221].

To recapitulate an earlier example [216], direct use of a salivary protein by $B$. burgdorferi may enhance its transmission as a benefit of down-regulation of the host immune system by that protein. Of particular note, Salp15 expression is enhanced in infected ticks compared to uninfected ticks, suggesting that the presence of $B$. burgdorferi modifies gene 
expression of I. scapularis. Ramamoorthi et al. [216] note that while the increased expression of Salp15 increases survival of B. burgdorferi, it also may increase that of I. scapularis, as immune responses raised against the spirochete may have negative consequences for successful feeding of the tick as well. Thus increased expression of Salp15 may be adaptive for I. scapularis, but only when in the presence of B. burgdorferi. This interaction is an example of coevolution between the microbe and vector that changes the tick-to-host transmission coefficient $\left(\beta_{\mathrm{T}-\mathrm{V}}\right)$. These results explain, in part, past unsuccessful attempts to infect laboratory animals by needle inoculation and again illustrate how ticks are not just "walking syringes" [218, 289].

\section{EVOLUTIONARY AND ECOLOGICAL CONTEXT FOR ADAPTATIONS OF LB SPIROCHETES}

In previous sections, I described molecular processes through which the spirochete, tick, and vertebrate interact. The implication was that if all the intricate molecular interactions were to favor the spirochete, it would have a $R_{0} \geq 1$ and be more likely to persist in future generations of vertebrate hosts and ticks. There was no explicit mention, however, of the population-level aspects of those interactions. Here, I highlight life history constraints, ecological interactions, and environmental factors that may influence the molecular interactions and thereby shape the fitness and population biology of LB spirochetes. I begin by reviewing one characteristic of the LB biocoenosis that may be the most important ecological factor contributing to the explosive emergence of LB in the USA. I then turn to other factors important not just to this parasite triangle, but to that of other vector-borne diseases as well.

\subsection{Generalists all around: implications for spread and impact on LB spirochete diversity}

The recent invasions of the $\mathrm{LB}$ tick and pathogen in the USA have been attributed largely to anthropogenic changes that favor the ecology of the LB system and have brought it into people's backyards and the woodlands where they recreate [14, 258]. Specifically, human-induced changes have increased the abundance of hosts for both ticks and B. burgdorferi and have brought wildlife and humans into closer contact. The generalist natures of the vector, the spirochete, and important vertebrate hosts subsequently have enabled the spread of LB.

Deer play a primary role in the persistence of $B$. burgdorferi in nature because they are the main determinate host on which adult I. scapularis feed and mate [292]. The growth and spread of $I$. scapularis tick populations are inextricably linked with that of deer. Deer populations in much of the eastern USA were nearly extirpated by the early 1900 s due to uncontrolled hunting, but then steadily rose in the twentieth century due to management. In the northeastern USA, the large-scale conversion of agricultural land to secondary succession forests fuelled the increase and expansion of deer populations from coastal refugia as well as that of other woodland creatures, many of which are reservoir species. Furthermore, the conversion of agricultural land and woodlands to suburban developments in the landscape created new deer habitat close to woodlands, replete with tasty ornamentals [14, 258].

Larval and nymphal $I$. scapularis feed on deer but also on numerous other species of mammals, birds, and lizards ([5] and references therein). Lawrie et al. [130] show that I. ricinus exhibits anti-complement activity against a range of mammalian and avian hosts, whereas the European I. hexagonus (a rather hostspecific tick) has a much narrower range. Thus, evasion of the host innate immune system may play a role not only in pathogen permissiveness, but in host selection as well [179]. Adaptations for a generalist feeding strategy increase the probability of securing a blood meal among the various forest habitats in which I. scapularis apparently can survive. While the quality of the blood meal with respect to survivorship and development of I. scapularis varies among species, its generalist nature contributes to its ability to invade novel ecological 
environments because it is not host-limited. (The same is undoubtedly true for I. ricinus and I. persculatus.)

While the full significance is yet unknown, it is interesting to note that in the USA, it is $B$. burgdorferi, a generalist LB spirochete species, that is spreading with $I$. scapularis. Many B. burgdorferi strains have been detected in several host taxa, and Hanincova et al. [97] hypothesize that it is the generalist nature of the bacterium that has enabled its contemporaneous population growth and geographic spread with that of the generalist vector [125]. Indeed, in the USA, where the LB system is dynamic, at least at the edges of its range, the generalist strategy of the tick further selects for generalist strains of B. burgdorferi. The reproductive success (i.e. $\mathrm{R}_{0}$ ) of various strains, which may have different consequences for disease in humans [55, 286, 294], the linkages among the ecology and evolution of $B$. burgdorferi and the disease and epidemiology of Lyme borreliosis.

Within endemic foci, the LB system and the ecosystem in which it exists may be relatively stable; and it is possible that vertebrate host specialization may occur over evolutionary time. This scenario may describe the historical situation in Europe, where several more hostspecialized LB spirochete species coexist that exhibit differential susceptibilities to host species-specific complement [122]. B. garinii and $B$. valaisaina appear to be avian-adapted [96]; B. afzelii tends to be mammal-adapted [95]; and $B$. lusitanae may be lizard-adapted $[147,234]$. In other words, for a given vertebrate host species, each of these species may have a higher $\mathrm{R}_{0}$ compared with $B$. burgdorferi. Indeed, researchers found that $B$. afzelii had greater fitness correlates (as quantified by $\beta_{\mathrm{T}-\mathrm{V}}$ and $\beta_{\mathrm{V}-\mathrm{T}}$ ) than $B$. burgdorferi when both were experimentally transmitted to rodent hosts, although the generality of the findings among host species remains to be tested [233]. Some researchers [28] propose that host specialization of B. burgdorferi strains in LB endemic areas of the northeastern USA also can be inferred, even if it is not as delineated as in Europe. With respect to geographic structure, this pattern of specialization can result in differential migration rates of the pathogen, resulting in, for example, a larger distribution for $B$. garinii compared to B. afzelii [252].

Evolutionary theories generally predict that ecological specialists should be favored (i.e. have higher $\mathrm{R}_{0}$ ) over generalists if resources are abundant and dependable [293]. Specialization can occur if adaptations to different habitats are antagonistic and thus result in functional trade-offs that limit the generalist's fitness in any one habitat [137]. However, the specialist strategy may carry a fitness cost, because the vectors are generalist ectoparasites that take few blood meals. In particular, specialization can have a negative effect on the tick-to-host transmission coefficient $\left(\beta_{\mathrm{T}-\mathrm{V}}\right)$ because the transmission of specialized genotypes, such as the rodent-adapted $B$. afzelii or the avianadapted $B$. valaisiana, will be precluded if ticks feed on non-permissive species.

How might host-specialization of LB spirochetes arise? At least two, non-mutually exclusive scenarios could lead to host specialization of B. burgdorferi, assuming that adaptations to various host species do not incur a tradeoff in fitness with any other aspect of the spirochete's life history. The first scenario is purely ecological, in that hosts that consistently present more frequent opportunities for feeding ticks will thereby act to select for certain strains. If host communities (species diversity and relative abundance) are relatively constant over time, host specialization of strains can be reinforced and increasing host specialization may occur, especially for hosts that contribute proportionately more to feeding and infecting ticks. If host communities are not stable (e.g. population cycling of voles or frequently disturbed communities), selection for more generalist strains may occur.

Another hypothesized mechanism by which host-specialization of spirochetes may occur is through subpopulations of ticks that vary genetically in host preference or feeding success. These tick populations would co-exist by feeding on different subsets of the vertebrate community as immatures, giving the overall appearance of a generalist strategy. This ecology may allow for increased host specialization of LB spirochete strains, as is seen within the I. uriae-driven system in 
which $B$. garinii cycles among sea bird species $[158,159,188]$. The convergence of adults on deer (or other larger mammals), however, may pose a challenge to this hypothesis if mate selection in LB vectors is random rather than assortative.

In addition to experiencing selection by host innate immune systems, the population structure of LB spirochetes may also be sculpted by host adaptive immunity. It has been hypothesized that adaptive immunity targeting ospC or antigens at linked loci play an important role in the population biology of LB spirochetes, through imposing negative frequency-dependent selection [24, 125, 213]. Theory predicts that the fitness of a common spirochete genotype relative to rare spirochete genotype will decrease within the host population as the number of immune hosts increase. Rare spirochete genotypes, therefore, will enjoy a fitness advantage that will then decline as they become more common. Frequency-dependent changes in fitness should result in quantitative changes in spirochete population structure over time and help maintain spirochete genetic variation. Indeed, significant year-to-year differences have been observed in the frequency distributions of $B$. burgdorferi genotypes in host-seeking ticks collected in the northeastern USA [213].

At this time, the role of host adaptive immunity on the observed fitness variation among strains is unknown. In particular, if expression of antigenic proteins varies between the tick and vertebrate environment, the selective effect of adaptive immunity may be somewhat damped, depending on the degree of crossprotection of antibodies to similar antigens (e.g. ospC types). The impact of frequencydependent selection also depends other factors, such as the relative use/abundance of various host species by vectors, the relative roles of innate and adaptive immunity, the longevity of the immune responses, as well as the longevity of the hosts themselves. Other non-host related factors selecting on unrelated traits may also indirectly influence the observed fitness variation among strains and thus the population structure of LB spirochetes.

\subsection{Abiotic factors and seasonality of host-seeking}

The seasonal activity of non-nidicolous hard ticks refers to their host-seeking activity, during which they climb atop vegetation and wait for a passing vertebrate host. As they host-seek, or "quest", ticks are vulnerable to a number of environmental factors, most importantly dessication. To avoid unfavorable environmental conditions (e.g. cold winters or hot summers) ticks host-seek only during certain periods of the year [256]. The initiation and termination of seasonal host-seeking activity seem to be photoperiod-dependent and vary among tick species and with climate $[19,66,90,126,256]$.

The shape of seasonal activity (= phenology) curves (Fig. 2) reflects a summation of factors that trigger the onset of host-seeking, the nutritional state of the ticks, and factors that remove them from the population of questing ticks. Ticks can host-seek while their energy reserves are sufficient [222]. The decline in the questing tick population over time is due to abiotic factors affecting questing behavior, mortality, as well as contacting vertebrate hosts [223, 224].

Different life stages of ticks can be active at different or similar times of the year. For example, in the northeastern USA the peak of seasonal activity of $I$. scapularis nymphs precedes that of larvae by at least one month, whereas there is much more overlap in the Upper Midwest [53]. In contrast, in Central Europe, seasonal activities of all life stages of I. ricinus are more synchronized with two general peaks of activity - one in spring and another in late summer/early autumn [90].

The degree of synchrony among life stages (given overlap in host species use) can influence the $\mathrm{R}_{0}$ of LB spirochetes. This is because the duration of infection among the host community is dynamic throughout the year. For example, as noted above, in the northeastern USA, P. leucopus, a relatively short-lived animal, plays an important role in the transmission cycles of $B$. burgdorferi. For P. leucopus to contribute to $B$. burgdorferi persistence in nature, $P$. leucopus must survive from when they are first infected by nymphs (late spring) until larvae emerge 
(late summer). As a result, the degree of synchrony of nymphal and larval host-seeking, which is determined by abiotic factors, can affect the efficiency of vertebrate-to-tick transmission $\left(\beta_{\mathrm{V}-\mathrm{T}}\right)$ and therefore $\mathrm{R}_{0}[125,181,185]$.

In addition to surviving, $P$. leucopus and other reservoir hosts must remain infectious until larvae emerge for the transmission cycle to be completed. In the laboratory, P. leucopus generally has been found to be infectious to xenodiagnostic larvae for much longer than their average natural lifespan [57] (although see [140] for an exception). Thus the traditional view holds that the infectivity of LB spirochetes to ticks lasts lifelong in reservoir hosts, and therefore, as long as hosts survive long enough, transmission to the next cohort of ticks will occur. Recently, however, it has been experimentally demonstrated that the duration of infectivity (r) can vary among strains, and for some strains, may decline significantly within a few weeks post-infection $[51,98]$. The intensity of vertebrate-to-tick transmission $\left(\beta_{\mathrm{V}-\mathrm{T}}\right)$ therefore can vary considerably among spirochete strains in P. leucopus, and perhaps with different strain-host species combinations. The variation in duration of infectivity may be a function of the ability of the strain to evade the vertebrate host's immune system, persist in the host, and remain transmissible to ticks.

In ecosystems with asynchronous feeding of nymphs and larvae, all else equal, natural selection will favor long-lived strains that are persistently infectious [185]. In other words, long-lived strains will tend to have higher $\mathrm{R}_{0}$ than short-lived strains, and over time, shortlived strains will go extinct unless other factors allow them to persist. Indeed, in questing nymphs collected in $P$. leucopus habitats of northeastern USA, where there is less synchrony, the infection prevalence of a B. burgdorferi genotype with short-term infectivity in P. leucopus (ospC-E = IGS9, an RST 3 type) is much lower than that of a genotype with longterm infectivity (ospC-A = IGS1, an RST 1 type) in the same host [27, 97, 287]. In contrast, in ecosystems with greater synchrony, shortlived strains might not be as disadvantaged. Furthermore, if there were tradeoffs in adaptations between transmission and persistence, short-lived strains may even possess a fitness advantage in areas with synchronous feeding $[181,185]$. These predictions are supported generally in a recent large-scale survey of questing I. scapularis nymphs in the USA: B. burgdorferi RST 1 strains are enriched in the Northeast, whereas RST2/3 types predominate in the Midwest [76]. It remains to be investigated how factors other than tick phenology (e.g. host communities and tick genetics) also may impact this difference in distribution of strains.

\subsection{Aggregation of ticks on hosts and non-systemic transmission}

In many host-parasite systems, parasites are distributed among hosts in an aggregated, rather than a random, manner [248]. Various hypotheses have been posed to explain the evolution of aggregated parasite distributions [6, 7], as well as how they result from heterogeneities in spatial distributions of hosts and parasites, behavior, immune status, etc. [291]. For vector-borne pathogens, aggregation of vectors on hosts obviously is important for pathogen maintenance, but the overall importance for LB spirochete persistence in nature is still undetermined.

Aggregation of ticks on hosts can increase vector survivorship and transmission efficiency, both of which may potentially increase $\mathrm{R}_{0}$. Increased numbers of cofeeding ticks result in greater repression of the host immune response due to proteins in tick saliva that have suppressive properties $[48,184]$, resulting in increased engorgement weights and feeding success $[78$, 134]. By increasing tick survivorship, the ratio of ticks to hosts $(\mathrm{N} / \mathrm{H})$ increases, thereby increasing $\mathrm{R}_{0}$. By helping ticks feed to repletion, aggregation may increase LB spirochete transmission from host to larvae $\left(\beta_{\mathrm{V}-\mathrm{T}}\right)$. In both USA and European studies [78, 134], there was a positive density-dependent effect on transmission rates of LB spirochetes from infected mice, but not from infected voles. Aggregation similarly should increase the probability of transmission from an infected nymph to a host $\left(\beta_{\mathrm{T}-\mathrm{V}}\right)$. It is unclear, however, how aggregation would affect $\mathrm{R}_{0}$ - both when co-feeding nymphs are infected with the same or different 
strains/species of LB spirochetes - in comparison to a random distribution.

Simultaneous aggregation of ticks on hosts may increase $R_{0}$ through non-systemic transmission; unlike for TBE virus, however, the importance of this pathway for LB spirochete maintenance is much less certain. Among the three post-embryonic life stages, larval ticks show the greatest level of aggregation. Thus, if larval ticks co-feed with infected adults and/ or with larvae infected through transovarial transmission, aggregation may increase $R_{0}$. Similarly, in areas where there is synchronous questing of nymphal and larval ticks, nonsystemic transmission could increase $\mathrm{R}_{0}$ during parts of the host-seeking season when fewer hosts are systemically infected. Non-systemic transmission does not require competent hosts, so it may increase $\mathrm{R}_{0}$ quantitatively as well as support less generalist strains - thereby contributing to maintenance of LB spirochete diversity. While the contribution of non-systemic transmission may be neglible or masked in systems that are not host-limited, this transmission route may be important for LB spirochete persistence in areas where reservoir hosts are not reliably abundant. For instance, non-systemic transmission permits the maintenance of endemic cycles of LB spirochetes in sheep in upland moorland habitats of United Kingdom, where alternative reservoir hosts are virtually absent [180, 222]. The features that facilitate non-systemic transmission are: (i) the immunomodulatory properties of tick saliva; (ii) aggregated distributions of ticks on the host population; and (iii) synchronized seasonal activity of different tick life stages.

In areas where there is asynchronous questing of nymphs and larvae, persistence of LB spirochetes depends on low recovery rate $(r)$ of hosts (i.e. selection of strains with high persistence) as well as the shared use by infectious nymphs and naïve larvae of the same individual hosts in the host community. Thus any mechanism that would increase the aggregation of naïve larval ticks on reservoir hosts that were previously parasitized by infected nymphs likely would increase $\mathrm{R}_{0}$ and LB spirochete persistence in nature.

\subsection{Interactions with other pathogens and tick endosymbionts}

I. ricinus-persulcatus complex ticks are infected with several microbial species, and it is unknown how they have shaped the population biology of LB spirochetes [267]. These other pathogens include viruses (e.g. tick-borne encephalitis fever virus), piroplasms (Babesia microti and $\mathrm{Ba}$. divergens), and other bacteria (e.g. Anaplasma phagocytophilum). I. ricinuspersulcatus complex ticks also can vector many other pathogenic bacteria including Rickettsia helvetica [68, 196] and Bartonella spp. [22, 45], but the epidemiological and enzootic importance of these microbes is yet to be determined.

Researchers have been interested in coinfection of ticks because of the potentially complicating effects for diagnosis and treatment [267]. Coinfections mainly have been observed for the pathogens that have higher individual prevalences. Thus, the coinfections that are most often observed in ticks are between LB spirochetes and A. phagocytophilum. Comparisons of single infection and coinfection prevalences among the various vector species, however, suggest that the two pathogens have independent transmission cycles [135, 241, 259, 267]. This inference of independent transmission cycles was supported by Levin and Fish [136] in their experimental investigation of the effect of coinfection on both the acquisition and transmission of B. burgdorferi and A. phagocytophilum in I. scapularis and P. leucopus. They found that infection with one pathogen did not hinder the acquisition of the other pathogen (i.e. $\beta_{\mathrm{T}-\mathrm{V}}$ ), and that co-infection did not affect the transmission efficiency of either pathogen to a susceptible tick (i.e. $\beta_{\mathrm{V}-\mathrm{T}}$ ). A recent analysis of infection data in the literature similarly suggests a lack of a strong effect of other LB spirochetes and A. phagocytophilum on spirochete transmission dynamics [82].

Besides the better known pathogens, researchers have found other microbes of unknown pathogenicity in I. ricinus-persulcatus complex ticks, e.g. B. miyamotoi, or a B. miyamotoi-like spirochete, more closely-related to 
relapsing fever spirochetes found in soft ticks $[167,232,247]$. As with other arthropods, rickettsial endosymbionts have been found [20, $163,208,240,259]$ and potentially may have important effects on transmission cycles [145]. Interestingly, Steiner et al. [259] found that coinfections of LB spirochetes with a spotted fever group rickettsia endosymbiont in male I. scapularis ticks occurred less often than predicted based on independent transmission expectations, although Moreno et al. [163] did not observe that among their samples. Further investigations of the microbial community ecology within I. ricinus-persulcatus complex ticks and their hosts may improve our understanding of the enzootiology of LB spirochetes and other pathogens [44].

\section{DISCUSSION: INCORPORATING AN ECOLOGICAL AND EVOLUTIONARY PERSPECTIVE INTO LB RESEARCH, MANAGEMENT AND CONTROL}

This review has highlighted many putative adaptations of LB spirochetes to their tick and vertebrate hosts, as well as biological traits of the vector and host that contribute to the maintenance of the LB biocoenosis. Increases in suitable habitat, the generalist natures of the ticks, spirochetes, and important vertebrate hosts, as well as the tick's high fecundity have enabled LB spirochete populations to spread rapidly in the USA and other areas of the world. How do these characteristics affect research concerning diagnosis, disease, vaccines and therapeutics as well as efficacy of LB management and control in the landscape?

The driving goal that motivates much of the current research to understand the adaptations of LB spirochetes for vectorial transmission is the quest for anti-pathogen and/or anti-tick vaccines. What gene target(s) would be the most effective for preventing transmission to humans or companion animals, or for breaking transmission cycles in nature by vaccinating wildlife? Can we predict the likelihood of escape mutants, and are some targets more prone to produce escape mutants? Will hosttargeted vaccines be more likely to select for vaccine-resistant strains compared to human vaccines, since humans are dead-end hosts for LB spirochetes and wildlife feed many more ticks than do humans? Can we predict consequences of escape mutants on disease manifestations for humans?

An approach that the US Department of Agriculture and Centers for Disease Control and Prevention have recently investigated for reducing LB risk in the USA is the use of host-targeted acaricides targeted at deer (to reduce the tick population) [211] and small rodent reservoirs (to divert and reduce immature ticks that are likely to become infected), respectively [56]. How will vector control affect both the abundance and local strain structure of LB spirochetes? For example, by targeting rodents with acaricide for killing larvae and nymphs, the approach selects for ticks and species (and/or strains) of LB spirochetes that feed on non-rodents - furthermore, what are the implications for disease as a consequence?

Climate change is predicted to expand the distribution of I. ricinus-persulcatus complex ticks and LB spirochetes into more northern regions [182]. If climate change affects the seasonality of the host-seeking phenologies, how will the strain structure and disease manifestations of LB spirochetes change regionally [185]? Climate change may also shift distributions of hosts. In the upper midwestern USA, researchers have documented a northern expansion of mammal species ranges, including that of $P$. leucopus, where it is has displaced many populations of the more northern $P$. maniculatus [168]. If host community composition and/or biodiversity changes as a function of landscape changes, climate change, or management, how will the strain structure and disease manifestations of LB spirochetes change?

Predicting the impact of environmental changes and management tools, such as vaccination or vector control, on LB risk and disease manifestations in humans requires a better understanding of the ecological and evolutionary processes that shape the population biology of LB spirochetes. A little more than a decade ago, researchers learned that transmission experiments using needle inoculations can lead to bizarre or incorrect inferences about 
transmission biology [218]. Today, as researchers become better able to investigate and manipulate molecular interactions in the parasite triangle, it is very important to consider the ecological and evolutionary context of the LB system to be more certain that research questions, experimental designs, and new control methods to reduce disease risk are meaningful, effective, and sustainable. Furthermore, with respect to a given spirochetal trait, researchers may find $\mathrm{R}_{0}$ useful as a framework to assess its adaptive value for the LB bacterium and therefore its potential as a target for control.

Acknowledgements. I gratefully acknowledge contributions by Klara Hanincova and Graham Hickling (who also assisted with figures) toward earlier drafts of this manuscript. I also thank Lorenza Beati, Olaf Kahl, Nicholas Ogden, Joseph Piesman, and two anonymous reviewers for providing many helpful comments and suggestions that have greatly improved the text. I also wish to recognize Klaus Kurtenbach, whose innovative work will continue to shape our understanding of LB spirochete ecology and population biology.

\section{REFERENCES}

[1] Alitalo A., Meri T., Ramo L., Jokiranta T., Heikkila T., Seppala I., et al., Complement evasion by Borrelia burgdorferi: serum-resistant strains promote $\mathrm{C} 3 \mathrm{~b}$ inactivation, Infect. Immun. (2001) 69: 3685-3691.

[2] Amore G., Tomassone L., Grego E., Ragagli C., Bertolotti L., Nebbia P., et al., Borrelia lusitaniae in immature Ixodes ricinus (Acari: Ixodidae) feeding on common wall lizards in Tuscany, central Italy, J. Med. Entomol. (2007) 44:303-307.

[3] Anderson J., Magnarelli L., Vertebrate host relationships and distribution of ixodid ticks (Acari: Ixodidae) in Connecticut, USA, J. Med. Entomol. (1980) 17:314-323.

[4] Anderson J., Johnson R., Magnarelli L., Hyde F., Identification of endemic foci of Lyme disease: isolation of Borrelia burgdorferi from feral rodents and ticks (Dermacentor variabilis), J. Clin. Microbiol. (1985) 22:36-38.

[5] Anderson J., Magnarelli L., Biology of Ticks, Infect. Dis. Clin. North Am. (2008) 22:195-215.

[6] Anderson R., May R., Regulation and stability of host-parasite population interactions. I. Regulatory processes, J. Anim. Ecol. (1978) 47:219-247.
[7] Anderson R., May R., Coevolution of hosts and parasites, Parasitology (1982) 85:411-426.

[8] Anderson R., May R., A framework for discussing the population biology of infectious diseases, in: Infectious diseases of humans - dynamics and control, Chapter 2, 1992, pp. 17-23.

[9] Anguita J., Ramamoorthi N., Hovius J., Das S., Thomas V., Persinski R., et al., Salp15, an Ixodes scapularis salivary protein, inhibits $\mathrm{CD} 4(+) \mathrm{T}$ cell activation, Immunity (2002) 16:849-859.

[10] Anguita J., Hedrick M., Fikrig E., Adaptation of Borrelia burgdorferi in the tick and the mammalian host, FEMS Microbiol. Rev. (2003) 27:493-504.

[11] Bacon M., Kugeler K., Mead P., Surveillance for Lyme disease - United States, 1992-2006, MMWR (2008) 57(SS10):1-9.

[12] Balashov Y., Bloodsucking ticks (Ixodidae) vectors of diseases of man and animals, Misc. Pub. Entomol. Soc. Am. (1972) 8:163-376.

[13] Baranton G., Postic D., Saintgirons I., Boerlin P., Piffaretti C., Assous M., Grimont P., Delineation of Borrelia burgdorferi sensu stricto, Borrelia garinii sp. nov., and group VS461 associated with Lyme borreliosis, Int. J. Syst. Bacteriol. (1992) 42:378-383.

[14] Barbour A., Fish D., The biological and social phenomenon of Lyme disease, Science (1993) 260: 1610-1616.

[15] Barbour A., Maupin G., Teltow G., Carter C., Piesman J., Identification of an uncultivable Borrelia species in the hard tick Amblyomma americanum: possible agent of a Lyme disease-like illness, J. Infect. Dis. (1996) 173:403-409.

[16] Barthold S., Feng S., Bockenstedt L., Fikrig E., Feen K., Protective and arthritis-resolving activity in sera of mice infected with Borrelia burgdorferi, Clin. Infect. Dis. (1997) 25:S9-S17.

[17] Barthold S., Hodzic E., Tunev S., Feng S., Antibody-mediated disease remission in the mouse model of Lyme borreliosis, Infect. Immun. (2006) 74: 4817-4825.

[18] Bell J., Stewart S., Wikel S., Resistance to tickborne Francisella tularensis by tick-sensitized rabbits: allergic klendusity, Am. J. Trop. Med. Hyg. (1970) 28: 876-880.

[19] Belozerov V., Diapause and biological rhythms in ticks, in: Obenchain F., Galun R. (Eds.), Physiology of ticks, Pergamon Press, 1982, pp. 469-500.

[20] Benson M., Gawronski J., Eveleigh D., Benson D., Intracellular symbionts and other bacteria associated with deer ticks (Ixodes scapularis) from 
Nantucket and Wellfleet, Cape Cod, Massachusetts, Appl. Environ. Microbiol. (2004) 70:616-620.

[21] Billeter S., Blanton H., Little S., Levy M., Breitschwerdt E., Detection of Rickettsia amblyommii in association with a tick bite rash, Vector Borne Zoonotic Dis. (2007) 7:607-610.

[22] Billeter S., Levy M., Chomel B., Breitschwerdt E., Vector transmission of Bartonella species with emphasis on the potential for tick transmission, Med. Vet. Entomol. (2008) 22:1-15.

[23] Bishopp F., Trembley H., Distribution and hosts of certain North American ticks, J. Parasitol. (1945) 31:1-53.

[24] Bockenstedt L., Hodzic E., Feng S., Bourrel K., Desilva A., Montgomery R., et al., Borrelia burgdorferi strain-specific OspC-mediated immunity in mice, Infect. Immun. (1997) 65:4661-4667.

[25] Bockenstedt L., Mao J., Hodzic E., Barthold S., Fish D., Detection of attenuated, noninfectious spirochetes in Borrelia burgdorferi-infected mice after antibiotic treatment, J. Infect. Dis. (2002) 186: 1430-1437.

[26] Boyard C., Vourc'h G., Barnounin J., The relationships between Ixodes ricinus and small mammal species at the woodland-pasture interface, Exp. Appl. Acarol. (2008) 44:61-76.

[27] Brisson D., Dykhuizen D., ospC diversity in Borrelia burgdorferi: different hosts are different niches, Genetics (2004) 168:713-722.

[28] Brisson D., Dykhuizen D., A modest model explains the distribution and abundance of Borrelia burgdorferi strains, Am. J. Trop. Med. Hyg. (2006) 74:615-622.

[29] Brossard M., Wikel S., Tick immunobiology, Parasitology (2004) 129:S161-176.

[30] Brunet L., Katavolos P., Spielman A., Telford S., Anti-OspA antibody reduces reservoir competence of mice for Borrelia burgdorferi, the agent of Lyme disease, Med. Vet. Entomol. (1997) 11:198-200.

[31] Bunikis J., Barbour A., Third Borrelia species in white-footed mice, Emerg. Infect. Dis. (2005) 11: 1150-1151.

[32] Burkot T., Piesman J., Wirtz R., Quantitation of the Borrelia burgdorferi outer surface protein A in Ixodes scapularis: fluctuations during the tick life cycle, doubling times, and loss while feeding, J. Infect. Dis. (1994) 170:883-889.

[33] Cabello F., Godfrey H., Newman S., Hidden in plain sight: Borrelia burgdorferi and the extracellular matrix, Trends Microbiol. (2007) 15:350-354.
[34] Canica M., Nato F., Dumerle L., Mazie J., Baranton G., Postic D., Monoclonal antibodies for identification of Borrelia afzelii sp. nov. associated with late cutaneous manifestations of Lyme borreliosis, Scand. J. Infect. Dis. (1993) 24:441-448.

[35] Carroll J., Schmidtmann E., Dispersal of blacklegged tick (Acari: Ixodidae) nymphs and adults at the woods-pasture interface, J. Med. Entomol. (1996) 33:554-558.

[36] Carroll J., Allen P., Hill D., Pound J.M., Miller J.A., George J.E., Control of Ixodes scapularis and Amblyomma americanum through use of the '4-poster' treatment device on deer in Maryland, Exp. Appl. Acarol. (2002) 28:289-296.

[37] Casjens S., Palmer N., Van Vugt R., Huang W., Stevenson B., Rosa P., et al., A bacterial genome in flux: the twelve linear and nine circular extrachromosomal DNAs in an infectious isolate of the Lyme disease spirochete Borrelia burgdorferi, Mol. Microbiol. (2000) 35:490-516.

[38] Charon N., Goldstein S., Genetics of motility and chemotaxis of a fascinating group of bacteria: The spirochetes, Annu. Rev. Genet. (2002) 36:47-73

[39] Chen S., Dumler J., Bakken J., Walker D., Identification of granulocytotropic Ehrlichia species as the etiologic agent of human disease, J. Clin. Microbiol. (1994) 32:589-595.

[40] Childs J., Pre-spillover prevention of emerging zoonotic diseases: what are the targets and what are the tools? Curr. Top. Microbiol. Immunol. (2007) 389-443.

[41] Chu C., Liu W., Jiang B., Wang D., Jiang W., Zhao Q., et al., Novel genospecies of Borrelia burgdorferi sensu lato from rodents and ticks in southwestern China, J. Clin. Microbiol. (2008) 46: 3130-3133.

[42] Clark K., Oliver J., James A., Durden L., Banks C., Prevalence of Borrelia burgdorferi sensu lato infection among rodents and host-seeking ticks in South Carolina, J. Med. Entomol. (2002) 39:198-206.

[43] Clark K., Hendricks A., Burge D., Molecular identification and analysis of Borrelia burgdorferi sensu lato in lizards in the southeastern United States, Appl. Environ. Microbiol. (2005) 71:2616-2625.

[44] Clay K., Fuqua C., Lively C., Wade M., Microbial community ecology of tick-borne human pathogens, in: Collinge S., Ray C. (Eds.), Disease ecology - community structure and pathogen dynamics, Chapter 4, 2006, pp. 41-57.

[45] Cotte V., Bonnet S., Le Rhun D., Le Naour E., Chauvin A., Boulouis H., et al., Transmission of 
Bartonella henselae by Ixodes ricinus, Emerg. Infect. Dis. (2008) 14:1074-1080.

[46] Craine N., Nuttall P., Marriott A., Randolph S., Role of grey squirrels and pheasants in the transmission of Borrelia burgdorferi sensu lato, the Lyme disease spirochaete, in the UK, Folia Parasitol. (1997) 44:155-160.

[47] Cross M., Buddle B., Aldwell F., The potential of oral vaccines for disease control in wildlife species, Vet. J. (2007) 174:472-480.

[48] Davidar P., Wilson M., Ribeiro J., Differential distribution of immature Ixodes dammini (Acari: Ixodidae) on rodent hosts, J. Parasitol. (1989) 75: 898-904.

[49] De Silva A., Telford S., Brunet L., Barthold S., Fikrig E., Borrelia burgdorferi OspA is an arthropodspecific transmission-blocking Lyme disease vaccine, J. Exp. Med. (1996) 183:271-275.

[50] De Silva A., Fikrig E., Arthropod- and hostspecific gene expression by Borrelia burgdorferi, J. Clin. Invest. (1997) 100:S3-S5.

[51] Derdakova M., Dudioak V., Brei B., Brownstein J., Schwartz I., Fish D., Interaction and transmission of two Borrelia burgdorferi sensu stricto strains in a tickrodent maintenance system, Appl. Environ. Microbiol. (2004) 70:6783-6788.

[52] Desilva A., Fikrig E., Borrelia burgdorferi genes selectively expressed in ticks and mammals, Parasitol. Today (1997) 13:267-270.

[53] Diuk-Wasser M., Gatewood A., Cortinas M., Yaremych-Hamer S., Tsao J., Kitron U., et al., Spatiotemporal patterns of host-seeking Ixodes scapularis nymphs (Acari: Ixodidae) in the United States, J. Med. Entomol. (2006) 43:166-176.

[54] Dizij A., Kurtenbach K., Clethrionomys glareolus, but not Apodemus flavicollis, acquires resistance to Ixodes ricinus L. the main European vector of Borrelia burgdorferi, Parasite Immunol. (1995) 17: 177-183.

[55] Dolan M., Piesman J., Schneider B., Schriefer M., Brandt K., Zeidner N., Comparison of disseminated and nondisseminated strains of Borrelia burgdorferi sensu stricto in mice naturally infected by tick bite, Infect. Immun. (2004) 72:5262-5266.

[56] Dolan M.C., Maupin G.O., Schneider B.S., Denatale C., Hamon N., Cole C., et al., Control of immature Ixodes scapularis (Acari: Ixodidae) on rodent reservoirs of Borrelia burgdorferi in a residential community of southeastern Connecticut, J. Med. Entomol. (2004) 41:1043-1054.

[57] Donahue J., Piesman J., Spielman A., Reservoir competence of white-footed mice for Lyme disease spirochetes, Am. J. Trop. Med. Hyg. (1987) 36:92-96.
[58] Eisen L., Eisen R., Lane R., Seasonal activity patterns of Ixodes pacificus nymphs in relation to climatic conditions, Med. Vet. Entomol. (2002) 16: 235-244.

[59] Eisen L., Lane R., Vectors of Borrelia burgdorferi sensu lato, in: Gray J., Kahl O., Lane R., Stanek G. (Eds.), Lyme borreliosis biology, epidemiology and control, Cabi Publishing, 2002, pp. 91-115.

[60] Eisen R., Mun J., Eisen L., Lane R., Life stagerelated differences in density of questing ticks and infection with Borrelia burgdorferi sensu lato within a single cohort of Ixodes pacificus (Acari: Ixodidae), J. Med. Entomol. (2004) 41:768-773.

[61] Falco R., Fish D., Horizontal movement of adult Ixodes dammini (Acari: Ixodidae) attracted to $\mathrm{CO}_{2}$ baited traps, J. Med. Entomol. (1991) 28:726-729.

[62] Feder H., Johnson B., O'connell S., Shapiro E., Steere A., Wormser G., Current concepts - A critical appraisal of "chronic Lyme disease", N. Engl. J. Med. (2007) 357:1422-1430.

[63] Fikrig E., Pal U., Chen M., Anderson J., Flavell R., OspB antibody prevents Borrelia burgdorferi colonization of Ixodes scapularis, Infect. Immun. (2004) 72:1755-1759.

[64] Fikrig E., Narasimhan S., Borrelia burgdorferi - Traveling incognito? Microbe Infect. (2006) 8: 1390-1399.

[65] Filippova N., Taxonomic aspects of the Lyme disease agent vector, Parassitologia (1990) 24:257267 (in Russian).

[66] Fish D., Population ecology of Ixodes dammini, in: Ginsberg H.S. (Ed.), Ecology and environment management of Lyme disease, Rutgers University Press, 1993, pp. 25-42.

[67] Foretz M., Postic D., Baranton G., Phylogenetic analysis of Borrelia burgdorferi sensu stricto by arbitrarily primed PCR and pulsed-field gel electrophoresis, Int. J. Syst. Bacteriol. (1997) 47:11-18.

[68] Fournier P., Allombert C., Supputamongkol Y., Caruso G., Brouqui P., Raoult D., An eruptive fever associated with antibodies to Rickettsia helvetica in Europe and Thailand, J. Clin. Microbiol. (2004) 42: 816-818.

[69] Francischetti I., Valenzuela J., Andersen J., Mather T., Ribeiro J., Ixolaris, a novel recombinant tissue factor pathway inhibitor (TFPI) from the salivary gland of the tick, Ixodes scapularis: Identification of factor $\mathrm{X}$ and factor $\mathrm{Xa}$ as scaffolds for the inhibition of factor VIIa/tissue factor complex, Blood (2002) 99:3602-3612.

[70] Francischetti I., Mather T., Ribeiro J., Cloning of a salivary gland metalloprotease and characterization 
of gelatinase and fibrin(ogen)lytic activities in the saliva of the Lyme disease tick vector Ixodes scapularis, Biochem. Biophys. Res. Commun. (2003) 305: 869-875.

[71] Francischetti I., Mather T.N., Ribeiro J.M., Penthalaris, a novel recombinant five-Kunitz tissue factor pathway inhibitor (TFPI) from the salivary gland of the tick vector of Lyme disease, Ixodes scapularis, Thromb. Haemost. (2004) 91:886-898.

[72] Francischetti I., Pham V., Mans B., Andersen J., Mather T.N., Lane R., Ribeiro J., The transcriptome of the salivary glands of the female western blacklegged tick Ixodes pacificus (Acari: Ixodidae), Insect Biochem. Mol. Biol. (2005) 35:1142-1161.

[73] Fraser C., Casjens S., Huang W., Sutton G., Clayton R., Lathigra R., et al., Genomic sequence of a Lyme disease spirochaete, Borrelia burgdorferi, Nature (1997) 390:580-586.

[74] Fukunaga M., Hamase A., Okada K., Nakao M., Borrelia tanukii sp. nov. and Borrelia turdae sp. nov. found from ixodid ticks in Japan: rapid species identification by $16 \mathrm{~S}$ rRNA gene-targeted PCR analysis, Microbiol. Immunol. (1996) 40:877-881.

[75] Garg R., Juncadella I.J., Ramamoorthi N.A., Ananthanarayanan S.K., Thomas V., Rincon M., et al., Cutting edge: CD4 is the receptor for the tick saliva immunosuppressor, Salp15, J. Immunol. (2006) 177: 6579-6583.

[76] Gatewood A., Liebman K., Vourc'h G., Bunikis J., Hamer S., Cortinas M., et al., Climate and tick seasonality predict Borrelia burgdorferi genotype distribution, Appl. Environ. Microbiol. (2009) 75: 2476-2483.

[77] Georgilis K., Peacocke M., Klempner M., Fibroblasts protect the Lyme disease spirochete, Borrelia burgdorferi, from ceftriaxone in vitro, J. Infect. Dis. (1992) 166:440-444.

[78] Gern L., Siegenthaler M., Hu C., Leubagarcia S., Humair P., Moret J., Borrelia burgdorferi in rodents (Apodemus flavicollis and A. sylvaticus) - duration and enhancement of infectivity for Ixodes ricinus ticks, Eur. J. Epidemiol. (1994) 10:75-80.

[79] Gern L., Estrada-Pena A., Frandsen F., Gray J.S., Jaenson T., Jongejan F., Kahl O., et al., European reservoir hosts of Borrelia burgdorferi sensu lato, Zentralbl. Bakteriol. (1998) 287: 196-204.

[80] Gillespie R., Dolan M., Piesman J., Titus R., Identification of an IL-2 binding protein in the saliva of the Lyme disease vector tick, Ixodes scapularis, J. Immunol. (2001) 166:4319-4326.

[81] Ginsberg H., Buckley P., Balmforth M., Zhioua E., Mitra S., Buckley F., Reservoir competence of native North American birds for the Lyme disease spirochete, Borrelia burgdorferi, J. Med. Entomol. (2005) 42:445-449.

[82] Ginsberg H., Potential effects of mixed infections in ticks on transmission dynamics of pathogens: comparative anlaysis of published records, Exp. Appl. Acarol. (2008) 46:29-41.

[83] Ginsberg H.S., Ewing C., O'connell A., Bosler E., Daley J., Sayre M., Increased population densities of Amblyomma americanum (Acari: Ixodidae) on Long Island, New York, J. Parasitol. (1991) 77: 493-495.

[84] Glockner G., Lehmann R., Romualdi A., Pradella S., Schulte-Spechtel U., Schilhabel M., et al., Comparative analysis of the Borrelia garinii genome, Nucleic Acids Res. (2004) 32:6038-6046.

[85] Glockner G., Schulte-Spechtel U., Schilhabel M., Felder M., Suehnel J., Wilske B., Platzer M., Comparative genome analysis: selection pressure on the Borrelia vls cassettes is essential for infectivity, BMC Genomics (2006) 7:6038-6044.

[86] Goddard J., Sumner J., Nicholson W., Paddock C., Shen J., Piesman J., Survey of ticks collected in Mississippi for Rickettsia. Ehrlichia, and Borrelia species, J. Vector Ecol. (2003) 28:184-189.

[87] Gould L., Nelson R., Griffith K., Hayes E., Piesman J., Mead P., Cartter M., Knowledge, attitudes, and behaviors regarding Lyme disease prevention among Connecticut residents, 1999-2004, Vector Borne Zoonotic Dis. (2008) 8:769-776.

[88] Grab D.J., Salim M., Chesney J., Bucala R., Lanners H.N., A role for peripheral blood fibrocytes in Lyme disease? Med. Hypotheses (2002) 59:1-10.

[89] Gray J., A carbon dioxide trap for prolonged sampling of Ixodes ricinus L. populations, Exp. Appl. Acarol. (1985) 1:35-44.

[90] Gray J., The development and seasonal activity of the tick Ixodes ricinus: a vector of Lyme borreliosis, Rev. Med. Vet. Entomol. (1991) 79:323-333.

[91] Gray J., The ecology of ticks transmitting Lyme borreliosis, Exp. Appl. Acarol. (1998) 22:249-258.

[92] Grimm D., Tilly K., Byram R., Stewart P., Krum J., Bueschel D., et al., Outer-surface protein $\mathrm{C}$ of the Lyme disease spirochete: A protein induced in ticks for infection of mammals, Proc. Natl. Acad. Sci. USA (2004) 101:3142-3147.

[93] Gritsun T., Lashkevich V., Gould E., Tick-borne encephalitis, Antiviral Res. (2003) 57:129-146.

[94] Hamer S., Tsao J., Walker E., Mansfield L., Foster E., Hickling G., Canines as sentinels for invading Ixodes scapularis and Borrelia burgdorferi 
in lower Michigan, J. Am. Vet. Med. Assoc. (2009) 70:49-56.

[95] Hanincova K., Schafer S., Etti S., Sewell H., Taragelova V., Ziak D., et al., Association of Borrelia afzelii with rodents in Europe, Parasitology (2003) 126:1-11.

[96] Hanincova K., Taragelova V., Koci J., Schafer S., Hails R., Ullmann A., et al., Association of Borrelia garinii and B. valaisiana with songbirds in Slovakia, Appl. Environ. Microbiol. (2003) 69: 2825-2830.

[97] Hanincova K., Kurtenbach K., Diuk-Wasser M., Brei B., Fish D., Epidemic spread of Lyme borreliosis, northeastern United States, Emerg. Infect. Dis (2006) 12:604-611.

[98] Hanincova K., Ogden N.H., Diuk-Wasser M., Pappas C.J., Iyer R., Fish D., et al., Fitness variation of Borrelia burgdorferi sensu stricto strains in mice, Appl. Environ. Microbiol. (2008) 74:153-157.

[99] Hartemink N.A., Randolph S.E., Davis S.A., Heesterbeek J.A.P., The basic reproduction number for complex disease systems: Defining $\mathrm{R}_{0}$ for tick-borne infections, Am. Nat. (2008) 171:743-754.

[100] Hellwage J., Meri T., Heikkila T., Alitalo A., Panelius J., Lahdenne P., et al., The complement regulator factor $\mathrm{H}$ binds to the surface protein OspE of Borrelia burgdorferi, J. Biol. Chem. (2001) 276: 8427-8435.

[101] Hodzic E., Feng S., Freet K., Barthold S., Borrelia burgdorferi population dynamics and prototype gene expression during infection of immunocompetent and immunodeficient mice, Infect. Immun. (2003) 71:5042-5055.

[102] Hodzic E., Feng S., Holden K., Freet K., Barthold S., Persistence of Borrelia burgdorferi following antibiotic treatment in mice, Antimicrob. Agents Chemother. (2008) 52:1728-1736.

[103] Hojgaard A., Eisen R., Piesman J., Transmission dynamics of Borrelia burgdorferi s.s. during the key third day of feeding by nymphal Ixodes scapularis (Acari: Ixodidae), J. Med. Entomol. (2008) 45: 732-736.

[104] Hovius J.W.R., Van Dam A.P., Fikrig E., Tickhost-pathogen interactions in Lyme borreliosis, Trends Parasitol. (2007) 23:434-438.

[105] Hovius J.W.R., De Jong M.A.W.P., Den Dunnen J., Litjens M., Fikrig E., Van Der Poll T., et al., Salp15 binding to DC-SIGN inhibits cytokine expression by impairing both nucleosome remodeling and mRNA stabilization, PLoS Pathog. (2008) 4:e31.
[106] Howe T., Laquier F., Barbour A., Organization of genes encoding 2 outer-membrane proteins of the Lyme disease agent Borrelia burgdorferi within a single transcriptional unit, Infect. Immun. (1986) 54: 207-212.

[107] Humair P., Gern L., Relationship between Borrelia burgdorferi sensu lato species, red squirrels (Sciurus vulgaris) and Ixodes ricinus in enzootic areas in Switzerland, Acta Trop. (1998) 69:213-227.

[108] Hynes W., Ceraul S., Todd S., Seguin K., Sonenshine D., A defensin-like gene expressed in the black-legged tick, Ixodes scapularis, Med. Vet. Entomol. (2005) 19:339-344.

[109] Iyer R., Hardham J., Wormser G., Schwartz I., Norris S., Conservation and heterogeneity of $v l_{s} E$ among human and tick Isolates of Borrelia burgdorferi, Infect. Immun. (2000) 68:1714-1718.

[110] Johns R., Sonenshine D., Hynes W., Response of the tick Dermacentor variabilis (Acari: Ixodidae) to hemocoelic inoculation of Borrelia burgdorferi (Spirochetales), J. Med. Entomol. (2000) 37:265-270.

[111] Johns R., Ohnishi J., Broadwater A., Sonenshine D., De Silva A., Hynes W., Contrasts in tick innate immune responses to Borrelia burgdorferi challenge: immunotolerance in Ixodes scapularis versus immunocompetence in Dermacentor variabilis (Acari: Ixodidae), J. Med. Entomol. (2001) 38: 99-107.

[112] Johns R., Sonenshine D., Hynes W., Identification of a defensin from the hemolymph of the American dog tick, Dermacentor variabilis, Insect Biochem. Mol. Biol. (2001) 31:857-865.

[113] Johnson R., Schmid G., Hyde F., Steigerwalt A., Brenner D., Borrelia burgdorferi sp. nov. etiologic agent of Lyme disease, Int. J. Syst. Bacteriol. (1984) 34:496-497.

[114] Kahl O., Janetzki-Mittmann C., Gray J., Jonas R., Stein J., De Boer R., Risk of infection with Borrelia burgdorferi sensu lato for a host in relation to the duration of nymphal Ixodes ricinus feeding and the method of tick removal, Zentralbl. Bakteriol. (1998) 287:41-52.

[115] Kahl O., Gern L., Eisen L., Lane R., Ecological research on Borrelia burgdorferi sensu lato: terminology and some methodological pitfalls, in: Gray J., Kahl O., Lane R., Stanek G. (Eds.), Lyme borreliosis, Cabi Publishing, 2002, pp. 29-46.

[116] Keesing F., Holt R., Ostfeld R., Effects of species diversity on disease risk, Ecol. Lett. (2006) 2006:4

[117] Keirans J., Hutcheson H., Durden L., Klompen J., Ixodes (Ixodes) scapularis (Acari: Ixodidae): 
Redescription of all active stages, distribution, hosts, geographical variation, and medical and veterinary importance, J. Med. Entomol. (1996) 33:297-318.

[118] Kraiczy P., Skerka C., Brade V., Zipfel P., Further characterization of complement regulatoracquiring surface proteins of Borrelia burgdorferi, Infect. Immun. (2001) 2001:12.

[119] Kraiczy P., Skerka C., Kirschfink M., Brade V., Zipfel P., Immune evasion of Borrelia burgdorferi by acquisition of human complement regulators FHL-1/ reconectin and Factor H, Eur. J. Immunol. (2001) 31:1674-1684.

[120] Kurtenbach K., Kampen H., Dizij A., Arndt S., Seitz H., Schaible U., Simon M., Infestation of rodents with larval Ixodes ricinus (Araci: Ixodidae) is an important factor in the transmission cycle of Borrelia burgdorferi sl in German woodlands, J. Med. Entomol. (1995) 32:807-817.

[121] Kurtenbach K., Carey D., Hoodless A., Nuttall P., Randolph S., Competence of pheasants as reservoirs for Lyme disease spirochetes, J. Med. Entomol. (1998) 35:77-81.

[122] Kurtenbach K., Sewell H., Ogden N., Randolph S., Nuttall P., Serum complement sensitivity as a key factor in Lyme disease ecology, Infect. Immun. (1998) 66:1248-1251.

[123] Kurtenbach K., De Michelis S., Etti S., Schafer S., Sewell H., Brade V., Kraiczy P., Host association of Borrelia burgdorferi sensu lato-the key role of host complement, Trends Microbiol. (2002) 10:74-79.

[124] Kurtenbach K., Schafer S., De Michelis S., Etti S., Sewell H., Borrelia burgdorferi sensu lato in the vertebrate host, in: Gray J., Kahl O., Lane R., Stanek G. (Eds.), Lyme borreliosis biology, epidemiology, and control, Cabi Publishing, 2002, pp. 117-148.

[125] Kurtenbach K., Hanincova K., Tsao J., Margos G., Fish D., Ogden N., Fundamental processes in the evolutionary ecology of Lyme borreliosis, Nat. Rev. Microbiol. (2006) 4:660-669.

[126] Lane R., Seasonal activity of two human-biting ticks, Calif. Agric. (1990) 44:23-25.

[127] Lane R., Piesman J., Burgdorfer W., Lyme borreliosis: relation of its causative agent to its vectors and hosts in North America and Europe, Annu. Rev. Entomol. (1991) 36:587-609.

[128] Lane R., Quistad G., Borreliacidal factor in the blood of the western fence lizard (Sceloporous occidentalis), J. Parasitol. (1998) 84:29-34.

[129] Lane R., Mun J., Eisen R., Eisen L., Western gray squirrel (Rodentia: Sciuridae): a primary reservoir host of Borrelia burgdorferi in California oak woodlands? J. Med. Entomol. (2005) 42:388-396.

[130] Lawrie C., Randolph S.E., Nuttall P., Ixodes ticks: serum species sensitivity of anticomplement activity, Exp. Parasitol. (1999) 93:207-214.

[131] Le Fleche A., Postic D., Girardet K., Peter O., Baranton G., Characterization of Borrelia lusitaniae sp. nov. by $16 \mathrm{~S}$ ribosomal DNA sequence analysis, Int. J. Syst. Bacteriol. (1997) 47: 921-925.

[132] Ledin K., Zeidner N., Ribeiro J., Biggerstaff B., Dolan M., Dietrich G., et al., Borreliacidal activity of saliva of the tick Amblyomma americanum, Med. Vet. Entomol. (2005) 19:90-95.

[133] Levin M., Levine J., Yang S., Howard P., Apperson C., Reservoir competence of the southeastern five-lined skink (Eumeces inexpectatus) and the green anole (Anolis carolinensis) for Borrelia burgdorferi, Am. J. Trop. Med. Hyg. (1996) 54:92-97.

[134] Levin M., Papero M., Fish D., Feeding density influences acquisition of Borrelia burgdorferi in larval Ixodes scapularis (Acari: Ixodidae), J. Med. Entomol. (1997) 34:569-572.

[135] Levin M., Des Vignes F., Fish D., Disparity in the natural cycles of Borrelia burgdorferi and the agent of human granulocytic ehrlichiosis, Emerg. Infect. Dis. (1999) 5:204-208.

[136] Levin M., Fish D., Acquisition of coinfection and simultaneous transmission of Borrelia burgdorferi and Ehrlichia phagocytophila by Ixodes scapularis ticks, Infect. Immun. (2000) 68:2183-2186.

[137] Levins R., Evolution in changing environments, Princeton University Press, 1968.

[138] Li X., Neelakanta G., Liu X., Beck D.S., Kantor F.S., Fish D., et al., Role of outer surface protein D in the Borrelia burgdorferi life cycle, Infect. Immun. (2007) 75:4237-4244.

[139] Li X., Pal U., Ramamoorthi N., Liu X., Desrosiers D., Eggers C., et al., The Lyme disease agent Borrelia burgdorferi requires BB0690, a Dps homologue, to persist within ticks, Mol. Microbiol. (2007) 63:694-710.

[140] Lindsay L., Barker I., Surgeoner G., Mcewen S., Campbell D., Duration of Borrelia burgdorferi infectivity in white-footed mice for the tick vector Ixodes scapularis under laboratory and field conditions in Ontario, J. Wildl. Dis. (1997) 33:766-775.

[141] Logiudice K., Ostfeld R., Schmidt K., Keesing F., The ecology of infectious disease: effects of host diversity and community composition on Lyme disease risk, Proc. Natl. Acad. Sci. USA (2003) 2003:567-571. 
[142] Lux R., Moter A., Shi W., Chemotaxis in pathogenic spirochetes: directed movement toward targeting tissues? J. Mol. Microbiol. Biotechnol. (2000) 2:355-364.

[143] Ma J., Hine P., Clough E., Fish D., Coughlin R., Beltz G., Shew M., Safety, efficacy, and immunogenecity of a recombinant Osp subunit canine Lyme disease vaccine, Vaccine (1996) 14:1366-1374.

[144] Ma Y., Sturrock A., Weis J., Intracellular localisation of Borrelia burgdorferi within human endothelial cell, Infect. Immun. (1991) 59:671-678.

[145] Macaluso K., Sonenshine D.E., Ceraul S., Azad A., Rickettsial infection in Dermacentor variabilis (Acari: Ixodidae) inhibits transovarial transmission of a second Rickettsia, J. Med. Entomol. (2002) 39:809-813.

[146] Madhav N., Brownstein J., Tsao J., Fish D., A dispersal model for the range expansion of blacklegged tick (Acari: Ixodidae), J. Med. Entomol. (2004) 41:842-852.

[147] Majlathova V., Majlath I., Derdakova M., Bronislava V., Pet'ko B., Borrelia lusitaniea and green lizards (Lacerta viridis), Karst Region, Slovakia, Emerg. Infect. Dis. (2006) 12:1895-1901.

[148] Marconi R., Samuels D., Garon C., Transcriptional analyses and mapping of the $O s p C$ gene in Lyme disease spirochetes, J. Bacteriol. (1993) 175: 926-932.

[149] Marconi R., Liveris D., Schwartz I., Identification of novel insertion elements, restriction fragment length polymorphism patterns, and discontinuous $23 \mathrm{~S}$ rRNA in Lyme disease spirochetes: phylogenetic analyses of rRNA genes and their intergenic spacers in Borrelia japonica sp. nov. and genomic group 21038 (Borrelia andersonii sp. nov.) isolates, J. Clin. Microbiol. (1995) 33: 2427-2434.

[150] Margos G., Gatewood A.G., Aanensen D.M., Hanincova K., Terekhova D., Vollmer S.A., et al., MLST of housekeeping genes captures geographic population structure and suggests a European origin of Borrelia burgdorferi, Proc. Natl. Acad. Sci. USA (2008) 105:8730-8735.

[151] Maritz-Olivier C., Stutzer C., Jongejan F., Neitz A., Gaspar A., Tick anti-hernostatics: targets for future vaccines and therapeutics, Trends Parasitol. (2007) 23: $397-407$.

[152] Masuzawa T., Takada N., Kudeken M., Fukui T., Yano Y., Ishiguro F., et al., Borrelia sinica sp. nov., a Lyme disease-related Borrelia species isolated in China, Int. J. Syst. Evol. Microbiol. (2001) 51: 1817-1824.
[153] Mather M., Telford S., Maclachlan A., Spielman A., Incompetence of catbirds as reservoirs for the Lyme disease spirochete, J. Parasitol. (1989) 75:66-69.

[154] Mather T., Wilson M., Moore S., Ribeiro J., Spielman A., Comparing the relative potential of rodents as reservoirs of the Lyme disease spirochete (Borrelia burgdorferi), Am. J. Epidemiol. (1989) 130:143-150.

[155] Mather T., Mather M., Intrinsic competence of 3 Ixodid ticks (Acari) as vectors of the Lyme disease spirochete, J. Med. Entomol. (1990) 27:646-650.

[156] Matuschka F., Fischer P., Musgrave K., Richter D., Spielman A., Hosts on which nymphal Ixodes ricinus most abundantly feed, Am. J. Trop. Med. Hyg. (1991) 44:100-107.

[157] Matuschka F., Heiler M., Eiffert H., Fischer P., Lotter H., Spielman A., Diversionary role of hoofed game in the transmission of Lyme disease spirochetes, Am. J. Trop. Med. Hyg. (1993) 48:693-699.

[158] Mccoy K., Boulinier T., Tirard C., Michalakis Y., Host specificity of a generalist parasite: genetic evidence of sympatric host races in the seabird tick Ixodes uriae, J. Evol. Biol. (2001) 14:395-405.

[159] Mccoy K., Boulinier T., Tirard C., Michalakis Y., Host-dependent genetic structure of parasite populations: Differential dispersal of seabird tick host races, Evolution (2003) 57:288-296.

[160] Mcdowell J., Sung S., Hu L., Marconi R., Evidence that the variable regions of the central domain of VlsE are antigenic during infection with Lyme disease spirochetes, Infect. Immun. (2002) 70:4196-4203.

[161] Monteiro R., Rezaie A., Ribeiro J., Francischetti I., Ixolaris: a factor Xa heparin-binding exosite inhibitor, Biochem. J. (2005) 387:871-877.

[162] Montgomery R., Nathanson M., Malawista S., The fate of Borrelia burgdorferi, the agent for Lyme disease, in mouse macrophages - destruction, survival, recovery, J. Immunol. (1993) 150:909-915.

[163] Moreno C., Moy F., Daniels T., Godfrey H., Cabello F., Molecular analysis of microbial communities identified in different developmental stages of Ixodes scapularis ticks from Westchester and Dutchess Counties, New York, Environ. Microbiol. (2006) 8:761-772.

[164] Moriarty T.J., Norman M.U., Colarusso P., Bankhead T., Kubes P., Chaconas G., Real-time high resolution $3 \mathrm{D}$ imaging of the lyme disease spirochete adhering to and escaping from the vasculature of a living host, PLoS Pathog. (2008) 4:e1000090. 
[165] Motaleb M., Corum L., Bono J., Elias A., Rosa P., Samuels D., Charon N., Borrelia burgdorferi periplasmic flagella have both skeletal and motility functions, Proc. Natl. Acad. Sci. USA (2000) 97: 10899-10904.

[166] Mukolwe S., Kocan A., Barker R., Kocan K., Murphy G., Attempted transmission of Borrelia burgdorferi (Spirochaetales: Spirochaetaceae) (JD1 Strain) by Ixodes scapularis (Acari: Ixodidae), Dermacentor variabilis, and Amblyomma americanum, J. Med. Entomol. (1992) 29:673-677.

[167] Mun J., Eisen R., Eisen L., Lane R., Detection of a Borrelia miyamotoi sensu lato relapsing-fever group spirochete from Ixodes pacificus in California, J. Med. Entomol. (2006) 43:120-123.

[168] Myers P., Lundrigan B., Hoffman S., Haraminac A., Seto S., Climate-induced changes in the small mammal communities of the northern Great Lakes, Glob. Chang. Biol. (2009) in press.

[169] Narasimhan S., Santiago F., Koski R., Brei B., Anderson J., Fish D., Fikrig E., Examination of the Borrelia burgdorferi transcriptome in Ixodes scapularis during feeding, J. Bacteriol. (2002) 184: 3122-3125.

[170] Narasimhan S., Montgomery R., Deponte K., Tschudi C., Marcantonio N., Anderson J., et al., Disruption of Ixodes scapularis anticoagulation by using RNA interference, Proc. Natl. Acad. Sci. USA (2004) 101:1141-1146.

[171] Narasimhan S., Sukumaran B., Bozdogan U., Thomas V., Liang X., Deponte K., et al., A tick antioxidant facilitates the Lyme disease agent's successful migration from the mammalian host to the arthropod vector, Cell Host Microbe (2007) 2:7-18.

[172] Nazareth R., Tomaz L., Ortiz-Costa S., Atella G., Ribeiro J., Francischetti I., Monteiro R., Antithrombotic properties of ixolaris, a potent inhibitor of the extrinsic pathway of the coagulation cascade, Thromb. Haemost. (2006) 96:7-13.

[173] Nazario S., Das S., De Silva A., Deponte K., Marcantonio N., Anderson J., et al., Prevention of Borrelia burgdorferi transmission in guinea pigs by tick immunity, Am. J. Trop. Med. Hyg. (1998) 58: $780-785$.

[174] Neelakanta G., Li X., Pal U., Liu X., Beck D.S., Deponte K., et al., Outer surface protein B is critical for Borrelia burgdorferi adherence and survival within Ixodes ticks, PLoS Pathog. (2007) 3: e33.

[175] Nordstrand A., Barbour A., Bergstrom S., Borrelia pathogenesis research in the post-genomic and post-vaccine era, Curr. Opin. Microbiol. (2000) 3: 36-92.
[176] Norman R., Bowers R., Begon M., Hudson P., Persistence of tick-borne virus in the presence of multiple host species: tick reservoirs and parasite mediated competition, J. Theor. Biol. (1999) 200: $111-118$.

[177] Norris S., Carter C., Howell J., Barbour A., Low-passage associated proteins of Borrelia burgdorferi B31 characterization and molecular cloning of OspD, a surface-exposed, plasmid-encoded lipoprotein, Infect. Immun. (1992) 60:4662-4672.

[178] Nosbisch L., Desilva A., Lack of detectable variation at Borrelia burgdorferi vlsE locus in ticks, J. Med. Entomol. (2007) 44:168-170.

[179] Nuttall P., Labuda M., Tick-host interactions: saliva-activated transmission, Parasitology (2004) 129: S177-S189.

[180] Ogden N., Nuttall P., Randolph S., Natural Lyme disease cycles maintained via sheep by cofeeding ticks, Parasitology (1997) 115:591-599.

[181] Ogden N., Bigras-Poulin M., O'callaghan C., Barker I., Kurtenbach K., Lindsay L., Charron D., Vector seasonality, host infection dynamics and fitness of pathogens transmitted by the tick Ixodes scapularis, Parasitology (2006) 134:209-227.

[182] Ogden N., Maarouf A., Barker I., Bigras-Poulin M., Lindsay L., Morshed M., et al., Climate change and the potential for range expansion of the Lyme disease vector Ixodes scapularis in Canada, Int. J. Parasitol. (2006) 36:63-70.

[183] Ogden N., Lindsay L., Hanincova K., Barker I., Bigras-Poulin M., Charron D., et al., Role of migratory birds in introduction and range expansion of Ixodes scapularis ticks and of Borrelia burgdorferi and Anaplasma phagocytophilum in Canada, Appl. Environ. Microbiol. (2008) 74:1780-1790.

[184] Ogden N.H., Kurtenbach K., Nutall P., Interstadial and infestation level-dependent variation in the transmission efficiency of Borrelia burgdorferi from mice to Ixodes ricinus ticks, Exp. Appl. Acarol. (1998) 22:367-372.

[185] Ogden N.H., Bigras-Poulin M., Hanincova K., Maarouf A., O'callaghan C.J., Kurtenbach K., Projected effects of climate change on tick phenology and fitness of pathogens transmitted by the North American tick Ixodes scapularis, J. Theor. Biol. (2008) 254:621-632.

[186] Ohnishi J., Piesman J., De Silva A., Antigenic and genetic heterogeneity of Borrelia burgdorferi populations transmitted by ticks, Proc. Natl. Acad. Sci. USA (2001) 98:670-675.

[187] Oliver J., Clark K., Chandler F., Tao L., James A., Banks C., et al., Isolation, cultivation, and 
characterization of Borrelia burgdorferi from rodents and ticks in the Charleston area of South Carolina, J. Clin. Microbiol. (2000) 38:120-124.

[188] Olsen B., Jaenson T., Noppa L., Bunikis J., Bergstrom S., A Lyme borreliosis cycle in seabirds and Ixodes uriae ticks, Nature (1993) 362:340-342.

[189] Pachner A., Basta J., Delaney E., Hulinska D., Localisation of Borrelia burgdorferi in murine Lyme borreliosis by electron microscopy, Am. J. Trop. Med. Hyg. (1995) 52:128-133.

[190] Pal U., De Silva A., Montgomery R., Fish D., Anguita J., Anderson J., et al., Attachment of Borrelia burgdorferi within Ixodes scapularis mediated by outer surface protein A, J. Clin. Invest. (2000) 106:561-569.

[191] Pal U., Fikrig E., Adaptation of Borrelia burgdorferi in the vector and vertebrate host, Microbes Infect. (2003) 5:659-666.

[192] Pal U., Li X., Wang T., Montgomery R., Ramamoorthi N., Desilva A., et al., TROSPA, an Ixodes scapularis receptor for Borrelia burgdorferi, Cell (2004) 119:457-468.

[193] Pal U., Yang X., Chen M., Bockenstedt L., Anderson J., Flavell R., et al., OspC facilitates Borrelia burgdorferi invasion of Ixodes scapularis salivary glands, J. Clin. Invest. (2004) 113:220-230.

[194] Pal U., Dai J., Li X., Neelakanta G., Luo P., Kumar M., Wang P., et al., A differential role for BB0365 in the persistence of Borrelia burgdorferi in mice and ticks, J. Infect. Dis. (2008) 197:148-155.

[195] Pal U., Wang P., Bao F., Yang X., Samanata S., Schoen R., et al., Borrelia burgdorferi basic membrane proteins $\mathrm{A}$ and $\mathrm{B}$ participate in the genesis of Lyme arthritis, J. Exp. Med. (2008) 205:133-141.

[196] Parola P., Tick-borne rickettsial diseases: emerging risks in Europe, Comp. Immunol. Microbiol. Infect. Dis. (2004) 27:297-304.

[197] Patrican L., Acquisition of Lyme disease spirochetes by cofeeding Ixodes scapularis ticks, Am. J. Trop. Med. Hyg. (1997) 57:589-593.

[198] Patrican L., Absence of Lyme disease spirochetes in larval progeny of naturally infected Ixodes scapularis (Acari: Ixodidae) fed on dogs, J. Med. Entomol. (1997) 34:52-55.

[199] Piesman J., Spielman A., Human babesiosis on Nantucket Island: prevalence of Babesia microti in ticks, Am. J. Trop. Med. Hyg. (1980) 29:742-746.

[200] Piesman J., Mather T., Sinsky R., Spielman A., Duration of tick attachment and Borrelia burdgorferi transmission, J. Clin. Microbiol. (1987) 25:557-558.
[201] Piesman J., Sinsky R., Ability of Ixodes scapularis, Dermacentor variabilis, and Amblyomma americanum (Acari, Ixodidae) to acquire, maintain, and transmit Lyme disease spirochetes (Borrelia burgdorferi), J. Med. Entomol. (1988) 25:336-339.

[202] Piesman J., Oliver J., Sinsky R., Growth kinetics of the Lyme disease spirochete (Borrelia burgdorferi) in vector ticks (Ixodes dammini), Am. J. Trop. Med. Hyg. (1990) 42:352-357.

[203] Piesman J., Standard system for infecting ticks (Acari: Ixodidae) with the Lyme disease spirochete, Borrelia burgdorferi, J. Med. Entomol. (1993) 30: 199-203.

[204] Piesman J., Happ C., Ability of the Lyme disease spirochete Borrelia burgdorferi to infect rodents and three species of human-biting ticks (blacklegged tick, American dog tick, lone star tick) (Acari: Ixodidae), J. Med. Entomol. (1997) 34: $451-456$

[205] Piesman J., Happ C., The efficacy of co-feeding as a means of maintaining Borrelia burgdorferi: a North American model system, J. Vector Ecol. (2001) 26:216-220

[206] Piesman J., Strategies for reducing Lyme borreliosis in North America, Int. J. Med. Microbiol. (2006) 296:(S1)17-22.

[207] Piesman J., Eisen L., Prevention of tick-borne diseases, Annu. Rev. Entomol. (2008) 53:323-343.

[208] Popov V., Korenberg E., Nefedova V., Han V., Wen J., Kovalevskii Y., et al., Ultrastructural evidence of the ehrlichial developmental cycle in naturally infected Ixodes persulcatus ticks in the course of coinfection with Rickettsia, Borrelia, and a flavivirus, Vector Borne Zoonotic Dis. (2007) 7:699-716.

[209] Postic D., Ras N., Lane R., Henderson M., Baranton G., Expanded diversity among California Borrelia isolates and description of Borrelia bissettii sp. nov (formerly Borrelia group DN127), J. Clin. Microbiol. (1998) 36:3497-3504.

[210] Postic D., Garnier M., Baranton G., Multilocus sequence analysis of atypical Borrelia burgdorferi sensu lato species - description of Borrelia californensis sp nov., and genospecies 1 and 2, Int. J. Med. Microbiol. (2007) 297:263-271.

[211] Pound J.M., Miller J.A., George J.E., Lemeilleur C.A., The '4-poster' passive topical treatment device to apply acaricide for controlling ticks (Acari: Ixodidae) feeding on white-tailed deer, J. Med. Entomol. (2000) 37:588-594.

[212] Preac-Mursic V., Weber K., Pfister H., Wilske B., Gross B., Baumann A., Prokop J., Survival of Borrelia 
burgdorferi in antibiotically treated patients with Lyme borreliosis, Infection (1989) 17:355-359.

[213] Qiu W., Bosler E., Campbell J., Ugine G., Wang I., Luft B., Dykhuizen D., A population genetic study of Borrelia burgdorferi sensu stricto from eastern Long Island, New York, suggested frequency-dependent selection, gene flow and host adaptation, Hereditas (1997) 127:203-216.

[214] Qiu W.G., Bruno J.F., Mccaig W.D., Xu Y., Livey I., Schriefer M.E., Luft B.J., Wide distribution of a high-virulence Borrelia burgdorferi clone in Europe and North America, Emerg. Infect. Dis. (2008) 14:1097-1104.

[215] Radolf J., Caimano M., The long strange trip of Borrelia burgdorferi outer-surface protein C, Mol. Microbiol. (2008) 69:1-4.

[216] Ramamoorthi N., Narasimhan S., Pal U., Bao F., Yang X., Fish D., et al., The Lyme disease agent exploits a tick protein to infect the mammalian host, Nature (2005) 436:573-577.

[217] Rand P.W., Lubelczyk C., Holman M.S., Lacombe E.H., Smith R., Abundance of Ixodes scapularis (Acari: Ixodidae) after the complete removal of deer from an isolated offshore island, endemic for Lyme disease, J. Med. Entomol. (2004) 41:779-784.

[218] Randolph S., Nuttall P., Nearly right or precisely wrong - natural versus laboratory studies of vector-borne diseases, Parasitol. Today (1994) 10: 458-462.

[219] Randolph S., Craine N., General framework for comparative quantitative studies of transmission of tick-borne diseases using Lyme borreliosis in Europe as an example, J. Med. Entomol. (1995) 32:765-777.

[220] Randolph S., Gern L., Nuttall P., Co-feeding ticks: Epidemiological significance for tick-borne pathogen transmission, Parasitol. Today (1996) 12: $472-479$.

[221] Randolph S., Miklisova D., Lysy J., Rogers D., Labuda M., Incidence from coincidence: patterns of tick infestations on rodents facilitate transmission of tick-borne encephalitis virus, Parasitology (1999) 118: 177-186.

[222] Randolph S., Storey K., Impact of microclimate on immature tick-rodent host interactions (Acari: Ixodidae): implications for parasite transmission, J. Med. Entomol. (1999) 1999:741-748.

[223] Randolph S., Tick ecology: processes and patterns behind the epidemiological risk exposed by ixodid ticks as vectors, Parasitology (2004) 129: S37-S65.
[224] Randolph S.E., Steele G., An experimental evaluation of conventional control measures against the sheep tick, Ixodes ricinus (L) (Acari: Ixodidae). 1. A unimodal seasonal activity pattern, Bull. Entomol. Res. (1985) 75:501-518.

[225] Ras N., Postic D., Foretz M., Baranton G., Borrelia burgdorferi sensu stricto, a bacterial species "Made in the USA"? Int. J. Syst. Bacteriol. (1997) 47:1112-1117.

[226] Ribeiro J., Makoul G., Levine J., Robinson D., Spielman A., Antihemostatic, anti-inflammatory, and immunosuppressive properties of the saliva of a tick, Ixodes dammini, J. Exp. Med. (1985) 161:332-344.

[227] Ribeiro J., Weis J., Telford S., Saliva of the tick Ixodes dammini inhibits neutrophil function, Exp. Parasitol. (1990) 70:382-388.

[228] Ribeiro J., Mather T., Ixodes scapularis: Salivary kininase activity is a metallo dipeptidyl carboxypeptidase, Exp. Parasitol. (1998) 89:213-221.

[229] Ribeiro J., Francischetti I., Role of arthropod saliva in blood feeding: Sialome and post-sialome perspectives, Annu. Rev. Entomol. (2003) 48:73-88.

[230] Ribeiro J., Alarcon-Chaidez F., Francischetti I., Mans B., Mather T., Valenzuela J., Wikel S., An annotated catalog of salivary gland transcripts from Ixodes scapularis ticks, Insect Biochem. Mol. Biol. (2006) 36:111-129.

[231] Richter D., Spielman A., Komar N., Matuschka F., Competence of American robins as reservoir hosts for Lyme disease spirochetes, Emerg. Infect. Dis. (2000) 6:133-138.

[232] Richter D., Schlee D., Matuschka F., Relapsing fever-like spirochetes infecting European vector tick of Lyme disease agent, Emerg. Infect. Dis. (2003) 9:697701.

[233] Richter D., Klug B., Spielman A., Matuschka F., Adaptation of diverse Lyme disease spirochetes in a natural rodent reservoir host, Infect. Immun. (2004) $72: 2442-2444$

[234] Richter D., Matuschka F.R., Perpetuation of the Lyme disease spirochete Borrelia lusitaniae by lizards, Appl. Environ. Microbiol. (2006) 72:4627-4632.

[235] Richter D., Postic D., Sertour N., Livey I., Matuschka F., Baranton G., Delineation of Borrelia burgdorferi sensu lato species by multilocus sequence analysis and confirmation of the delineation of Borrelia spielmanii sp. nov., Int. J. Syst. Evol. Microbiol. (2006) 56:873-881.

[236] Rosa P., Tilly K., Stewart P., The burgeoning molecular genetics of the Lyme disease spirochaete, Nat. Rev. Microbiol. (2005) 3:129-143. 
[237] Rudenko N., Golovchenko M., Grubhoffer L., Oliver J.H. Jr., Borrelia carolinensis sp. nov. a new (14th) member of the Borrelia burgdorferi sensu lato complex from the southeastern region of the United States, J. Clin. Microbiol. (2009) 47:134-141.

[238] Ryder J., Pinger R., Glancy T., Inability of Ixodes cookei and Amblyomma americanum nymphs (Acari: Ixodidae) to transmit Borrelia burgdorferi, J. Med. Entomol. (1992) 29:525-530.

[239] Sanders S.F., Oliver J., Evaluation of Ixodes scapularis, Amblyomma americanum, and Dermacentor variabilis (Acari: Ixodidae) from Georgia as vectors of a Florida strain of the Lyme disease spirochete, Borrelia burgdorferi, J. Med. Entomol. (1995) 32:402-406.

[240] Schabereiter-Gurtner C., Lubitz W., Rolleke S., Application of broad-range 16S rRNA PCR amplification and DGGE fingerprinting for detection of tickinfecting bacteria, J. Microbiol. Methods (2003) 52:251-260.

[241] Schauber E., Gertz S., Maple W., Ostfeld R., Coinfection of blacklegged ticks (Acari: Ixodidae) in Dutchess County, New York, with the agents of Lyme disease and human granulocytic ehrlichiosis, J. Med. Entomol. (1998) 35:901-903.

[242] Scheckelhoff M.R., Telford S.R., Wesley M., $\mathrm{Hu}$ L.T., Borrelia burgdorferi intercepts host hormonal signals to regulate expression of outer surface protein A, Proc. Natl. Acad. Sci. USA (2007) 104:7247-7252.

[243] Schulze T.L., Bowen G., Bosler E., Lakat M., Parkin W., Altman R., et al., Amblyomma americanum - a potential vector of Lyme disease in New Jersey, Science (1984) 224:601-603.

[244] Schwan T., Burdorfer W., Garon C., Changes in infectivity and plasmid profile of the Lyme disease spirochete, Borrelia burgdorferi, as a result of in vitro cultivation, Infect. Immun. (1988) 56:1831-1836.

[245] Schwan T., Piesman J., Golde W., Dolan M., Rosa P., Induction of an outer surface protein on Borrelia burgdorferi during tick feeding, Proc. Natl. Acad. Sci. USA (1995) 92:2909-2913.

[246] Schwan T., Piesman J., Temporal changes in outer surface proteins A and C of the Lyme diseaseassociated spirochete, Borrelia burgdorferi, during the chain of infection in ticks and mice, J. Clin. Microbiol. (2000) 38:382-388.

[247] Scoles G.A., Papero M., Beati L., Fish D., A relapsing fever group spirochete transmitted by Ixodes scapularis ticks, Vector Borne Zoonotic Dis. (2001) 1:21-34.
[248] Shaw D., Grenfell B., Dobson A., Patterns of macroparasite aggregation in wildlife host populations, Parasitology (1998) 117:597-610.

[249] Shi Y., Xu Q., Seemanapalli S.V., Mcshan K., Liang F.T., The dbpBA locus of Borrelia burgdorferi is not essential for infection of mice, Infect. Immun. (2006) 74:6509-6512.

[250] Shih C., Chao L., Yu C., Chemotactic migration of the Lyme disease spirochete (Borrelia burgdorferi) to salivary gland extracts of vector ticks, Am. J. Trop. Med. Hyg. (2002) 66:616-621.

[251] Singh S., Girschick H., Molecular survival strategies of the Lyme disease spirochete Borrelia burgdorferi, Lancet (2004) 4:575-583.

[252] Smith R., Bin Muzaffar S., Lavers J., Lacombe E., Cahill B., Lubelczyk C., et al., Borrelia garinii in seabird ticks (Ixodes uriae), Atlantic coast, North America, Emerg. Infect. Dis. (2006) 12:1212-1909.

[253] Soares C., Zeidner N., Beard C., Dolan M., Dietrich G., Piesman J., Kinetics of Borrelia burgdorferi infection in larvae of refractory and competent tick vectors, J. Med. Entomol. (2006) 43:61-67.

[254] Solberg V., Miller J.A., Hadfield T., Burge R., Schech J., Pound J.M., Control of Ixodes scapularis (Acari: Ixodidae) with topical self-application of permethrin by white-tailed deer inhabiting NASA, Beltsville, Maryland, J. Vector Ecol. (2003) 28: 117-134.

[255] Sonenshine D.E., Biology of Ticks. Volume 1, Oxford University Press, 1991.

[256] Sonenshine D.E., The biology of tick vectors of human disease, in: Goodman L., Dennis D., Sonenshine D.E. (Eds.), Tick-borne diseases of humans, 2005, pp. 12-36.

[257] Sonenshine D.E., Hynes W.L., Molecular characterization and related aspects of the innate immune response in ticks, Front. Biosci. (2008) 13:7046-7063.

[258] Steere A., Coburn J., Glickstein L., The emergence of Lyme disease, J. Clin. Invest. (2004) 113:1093-1101.

[259] Steiner F., Pinger R., Vann C., Grindle N., Civitello D., Clay K., Fuqua C., Infection and coinfection rates of Anaplasma phagocytophilum variants, Babesia spp., Borrelia burgdorferi, and the rickettsial endosymbiont in Ixodes scapularis (Acari: Ixodidae) from sites in Indiana, Maine, Pennsylvania, and Wisconsin, J. Med. Entomol. (2008) 2008:2.

[260] Stevenson B., El-Hage N., Hines M., Miller J., Babb K., Differential binding of host complement inhibitor factor $\mathrm{H}$ by Borrelia burgdorferi Erp surface 
proteins: a possible mechanism underlying the expansive host range of Lyme disease spirochetes, Infect. Immun. (2002) 70:491-497.

[261] Stewart P., Byram R., Grimm D., Tilly K., Rosa P., The plasmids of Borrelia burgdorferi: essential genetic elements of a pathogen, Plasmid (2005) 53: $1-13$.

[262] Stewart P., Wang X., Bueschel D., Clifton D., Grimm D., Tilly K., et al., Delineating the requirement for the Borrelia burgdorferi virulence factor OspC in the mammalian host, Infect. Immun. (2006) 74: 3547-3553.

[263] Stewart P.E., Bestor A., Cullen J.N., Rosa P.A., A tightly regulated surface protein of Borrelia burgdorferi is not essential to the mouse-tick infectious cycle, Infect. Immun. (2008) 76:1970-1978.

[264] Straubinger R., PCR-based quantification of Borrelia burgdorferi organisms in canine tissues over a 500-day postinfection period, J. Clin. Microbiol. (2000) 38:2191-2199.

[265] Straubinger R., Straubinger A., Summers B., Jacobson R., Status of Borrelia burgdorferi infection after antibiotic treatment and the effects of corticosteroids: An experimental study, J. Infect. Dis. (2000) 181:1069-1081.

[266] Swanson K.I., Norris D.E., Detection of Borrelia burgdorferi DNA in lizards from southern Maryland, Vector Borne Zoonotic Dis. (2007) 2007:42-49.

[267] Swanson S.J., Neitzel D., Reed K.D., Belongia E.A., Coinfections acquired from Ixodes ticks, Clin. Microbiol. Rev. (2006) 19:708-727.

[268] Talleklint L., Jaenson T., Maintenance by hares of European Borrelia burgdorferi in ecosystems without rodents, J. Med. Entomol. (1993) 30:273-276.

[269] Telford S., Mather T., Moore S., Wilson M., Spielman A., Incompetence of deer as reservoirs of the Lyme disease spirochete, Am. J. Trop. Med. Hyg. (1988) 39:105-109.

[270] Telford S., Spielman A., Competence of a rabbit-feeding Ixodes (Acari: Ixodidae) as a vector of the Lyme disease spirochete, J. Med. Entomol. (1989) 26:118-121.

[271] Telford S., Mather T., Adler G., Spielman A., Short-tailed shrews as reservoirs of the agents of Lyme disease and human babesiosis, J. Parasitol. (1990) 76:681-683.

[272] Telford S., Armstrong P., Katavolos P., Foppa I., Garcia A., Wilson M., Spielman A., A new tick-borne encephalitis-like virus infecting New England deer ticks, Ixodes dammini, Emerg. Infect. Dis. (1997) 3:165-170.

[273] Teltow G., Fournier J., Rawlings J., Isolation of Borrelia burdorferi from arthropods collected in Texas, Am. J. Trop. Med. Hyg. (1991) 44:469-474.

[274] Terekhova D., Iyer R., Wormser G.P., Schwartz I., Comparative genome hybridization reveals substantial variation among clinical isolates of Borrelia burdorferi sensu stricto with different pathogenic properties, J. Bacteriol. (2006) 188:6124-6134.

[275] Tilly K., Krum J., Bestor A., Jewett M., Grimm D., Bueschel D., et al., Borrelia burgdorferi OspC protein required exclusively in a crucial early stage of mammalian infection, Infect. Immun. (2006) 74: 3554-3564.

[276] Tilly K., Rosa P.A., Stewart P.E., Biology of infection with Borrelia burgdorferi, Infect. Dis. Clin. North Am. (2008) 22:217-234.

[277] Troughton D., Levin M., Life cycles of seven ixodid tick species (Acari: Ixodidae) under standardized laboratory conditions, J. Med. Entomol. (2007) 2007:732-740.

[278] Tsao J., Wootton J., Bunikis J., Luna M., Fish D., Barbour A., An ecological approach to preventing human infection: Vaccinating wild mouse reservoirs intervenes in the Lyme disease cycle, Proc. Natl. Acad. Sci. USA (2004) 101:18159-18164.

[279] Tyson K., Elkins C., Patterson H., Fikrig E., De Silva A., Biochemical and functional characterization of Salp20, an Ixodes scapularis tick salivary protein that inhibits the complement pathway, Insect Mol. Biol. (2007) 16:469-479.

[280] Urioste S., Hall L., Telford S., Titus R., Saliva of the Lyme disease vector, Ixodes dammini, blocks cell activation by a nonprostaglandin $\mathrm{E}(2)$-dependent mechanism, J. Exp. Med. (1994) 180:1077-1085.

[281] Valenzuela J., Francischetti I., Pham V., Garfield M., Mather T., Ribeiro J., Exploring the sialome of the tick Ixodes scapularis, J. Exp. Biol. (2002) 205:2843-2864.

[282] Vourc'h G., Marmet J., Chassagne M., Bord S., Chapuis J., Borrelia burgdorferi sensu lato in Siberian chipmunks (Tamias sibiricus) introduced in suburban forests in France, Vector Borne Zoonotic Dis. (2007) 7:637-641.

[283] Walker E., Smith T., Dewitt J., Beaudo D., Mclean R., Prevalence of Borrelia burgdorferi in hostseeking ticks (Acari: Ixodidae) from a Lyme disease endemic area in northern Michigan, J. Med. Entomol. (1994) 31:524-528. 
[284] Wallich R., Pattathu J., Kitiratschky V., Brenner C., Zipfel P., Brade V., et al., Identification and functional characterization of complement regulator-acquiring surface protein 1 of the Lyme disease spirochetes Borrelia afzelii and Borrelia garinii, Infect. Immun. (2005) 73:2351-2359.

[285] Wang G., Van Dam A., Le Fleche A., Postic D., Peter O., Baranton G., et al., Genetic and phenotypic analysis of Borrelia valaisiana sp. nov. (Borrelia genomic groups VS116 and M19), Int. J. Syst. Bacteriol. (1997) 47:926-932.

[286] Wang G., Ojaimi C., Iyer R., Saksenberg V., Mcclain S., Wormser G., Schwartz I., Impact of genotypic variation of Borrelia burgdorferi sensu stricto on kinetics of dissemination and severity of disease in $\mathrm{C} 3 \mathrm{H} / \mathrm{HeJ}$ mice, Infect. Immun. (2001) 69:4303-4312.

[287] Wang I., Dykhuizen D., Qin W., Dunn J., Bosler E., Luft B.J., Genetic diversity of OspC in a local population of Borrelia burgdorferi sensu stricto, Genetics (1999) 151:15-30.

[288] Wikel S., Tick modulation of host cytokines, Exp. Parasitol. (1996) 84:304-309.

[289] Wikel S., Tick modulation of host immunity: an important factor in pathogen transmission, Int. J. Parasitol. (1999) 29:851-859.

[290] Willadsen P., Jongejan F., Immunology of the tick-host interaction and the control of ticks and tickborne diseases, Parasitol. Today (1999) 15:258-262.

[291] Wilson K., Bjornstad O., Dobson A., Merler S., Poglayen G., Randolph S.E., et al., Heterogeneities in macroparasite infections: patterns and processes, in: Hudson P., Rizzoli A., Grenfell B., Heesterbeek H., Dobson A. (Eds.), The ecology of wildlife diseases, Oxford University Press, 2002, pp. 6-44.

[292] Wilson M., Adler G., Spielman A., Correlation between abundance of deer and that of the deer tick, Ixodes dammini (Acari: Ixodidae), Ann. Entomol. Soc. Am. (1985) 78:172-176.

[293] Woolhouse M., Taylor L., Haydon D., Population biology of multihost pathogens, Science (2001) 292:1101-1112.

[294] Wormser G., Liveris D., Nowakowski J., Nadelman R., Cavaliere L., Mckenna D., et al., Association of specific subtypes of Borrelia burgdorferi with hematogenous dissemination in early Lyme disease, J. Infect. Dis. (1999) 180:720-725.

[295] Wormser G., Dattwyler R., Shapiro E., Halperin J., Steere A., Klempner M., et al., The clinical assessment, treatment, an prevention of Lyme disease, human granulocytic analplasmosis, and babesiosis: clinical practice guidelines by the Infectious Diseases
Society of America, Clin. Infect. Dis. (2006) 43: 1089-1134.

[296] Xu G., Fang Q., Keirans J., Durden L., Molecular phylogenetic analyses indicate that the Ixodes ricinus complex is a paraphyletic group, J. Parasitol. (2003) 89:452-457.

[297] Xu Q., Mcshan K., Liang F., Essential protective role attributed to the surface lipoproteins of Borrelia burgdorferi against innate defences, Mol. Microbiol. (2008) 69:15-29.

[298] Xu Y., Kodner C., Coleman L., Johnson R., Correlation of plasmids with infectivity of Borrelia burgdorferi sensu stricto type strain B31, Infect. Immun. (1996) 64:3870-3876.

[299] Yang X., Pal U., Alani S., Fikrig E., Norgard M., Essential role for OspA/B in the life cycle of the Lyme disease spirochete, J. Exp. Med. (2004) 199:641-648.

[300] Zeidner N., Dreitz M., Belasco D., Fish D., Suppression of acute Ixodes scapularis-induced Borrelia burgdorferi infection using tumor necrosis factoralpha, interleukin-2, and interferon-gamma, J. Infect. Dis. (1996) 173:187-195.

[301] Zhang J., Norris S., Genetic variation of the Borrelia burgdorferi gene $v l s E$ involves cassettespecific, segmental gene conversion, Infect. Immun. (1998) 66:3698-3704.

[302] Zhang X., Meltzer M., Pena C., Hopkins A., Wroth L., Fix A., Economic impact of Lyme disease, Emerg. Infect. Dis. (2006) 12:653-660.

\section{Appendix}

\section{$R_{0}$ : A FRAMEWORK TO ASSESS ADAPTIVE STRATEGIES OF LB SPIROCHETES TO VECTORIAL TRANSMISSION}

\section{$R_{0}$ : A MEASURE OF PATHOGEN FITNESS}

At the heart of the parasite triangle (Fig. 1) is the fitness of the pathogen, quantified by the basic reproductive number, $\mathrm{R}_{0} . \mathrm{R}_{0}$ is defined as the number of secondary infections resulting from a primary infected individual that has been introduced into a population of completely susceptible individuals [8]. If $\mathrm{R}_{0}=1$, then the pathogen is replacing itself and is said to be endemic in the host population. If $\mathrm{R}_{0}>1$, many secondary cases result from the primary case, 
and an epizootic results. If $\mathrm{R}_{0}<1$, then the primary case fails to infect any susceptible individuals, and the pathogen goes extinct (epidemic fadeout). Vector-borne pathogens have developed a wide range of adaptations in each of the triangle components that make them better suited to each of their tick and vertebrate host environments, which also sit in the larger external environment. Thus, to assess the contribution of adaptations to the pathogen, it is essential to examine each in the context of the overall life cycle.

\section{$R_{0}$ EQUATION FOR THE AGENTS OF LB}

Randolph and Craine [219] first developed the mathematical expression for $\mathrm{R}_{0}$ for a tickborne pathogen. They point out that in contrast to an expression developed for mosquito-borne pathogens, the calculation of $\mathrm{R}_{0}$ for tick-borne pathogens must take into account the long-lived nature of ticks relative to their hosts by including tick population dynamics. The expression they developed for $\mathrm{R}_{0}$ for $\mathrm{LB}$ spirochetes is:

$$
\begin{aligned}
R_{0}= & \left(N f \beta_{V-T} \beta_{T-T} \beta_{T-V} p^{n} F\right) \\
& / H(r+h),
\end{aligned}
$$

where $N / H$ is the ratio of ticks to vertebrate hosts; $\mathrm{f}$ is the combined probability of a tick contacting and feeding successfully on a host; $\beta_{V-T}$ is the transmission coefficient for LB spirochetes from an infected vertebrate to a tick; $\beta_{T-T}$ is the transstadial transmission coefficient through the molt from one life stage to the next one; $\beta_{\mathrm{T}-\mathrm{V}}$ is the transmission coefficient for LB spirochetes from an infected tick to a vertebrate; $p$ is the tick's daily survival probability; $n$ is the extrinsic incubation period; $F$ is the vector's fecundity; $r$ is the host's daily recovery rate (i.e. the loss of the ability to transmit spirochetes to susceptible ticks); and $h$ is the host's daily mortality rate. From this expression, one can see that any factors that increase the values in the numerator or decrease the values in the denominator will increase $\mathrm{R}_{0}$ and its persistence through time. In particular, because it contains an exponential term, $p^{n}$ affects $\mathrm{R}_{0}$ profoundly.

One also can see how genetic variation among strains within a population could result in different phenotypes that will experience different fitnesses, subsequently resulting in strainspecific $R_{0}$. For each strain, $R_{0}$ will be a sum of that from each host species. Then at the population level, $\mathrm{R}_{0}$ for a local population of LB spirochetes is equal to the sum of the individual $R_{0}$ weighted by the relative abundance of each genotype.

\section{ADAPTATIONS AND $\mathbf{R}_{\mathbf{0}}$}

$\mathrm{R}_{0}$ provides a framework to assess the adaptive value of a particular phenotype of the spirochete. Any heritable trait that increases $R_{0}$ for LB spirochetes is considered an adaptation. For example, if $\mathrm{R}_{0}$ were increased because a spirochete possesses a variation of an outer surface protein molecule that allows it to evade a vertebrate host's immune system better than other variants, that variant would be hypothesized to be an adaptation. It is important to remember that (i) it is the environment that determines which variations are adaptive, and likewise, (ii) traits that are adaptive in one environment may not be as adaptive, or may even be maladaptive, in another. 